\title{
Evidence of a Free-Space Ion Acceleration Layer in the Plume of a Quad Confinement Plasma Source
}

\author{
Andrea Lucca Fabris, ${ }^{1, a)}$ Christopher V. Young, ${ }^{2, b)}$ Aaron Knoll, ${ }^{3}$ Emmanuelle K. Rosati Azevedo, ${ }^{3}$ and Mark A. \\ Cappelli $^{2}$ \\ 1) Surrey Space Centre, University of Surrey, Guildford GU2 7XH, UK \\ ${ }^{2)}$ Mechanical Engineering Department, Stanford University, Stanford, CA 94305 \\ ${ }^{3)}$ Department of Aeronautics, Imperial College London, London, UK, SW7 $2 A Z$
}

The Quad Confinement Plasma Source is a novel plasma device developed for space propulsion applications, whose core is an $E \times B$ discharge with open electron drift. The magnetic field is produced by independently powered electromagnets able to generate different magnetic field topologies with the ultimate aim of manipulating the ion flow field for achieving thrust vectoring. In this work, we map the ion velocity in the plasma ejected from the Quad Confinement Thruster with different magnetic configurations using nonintrusive Laser-Induced Fluorescence diagnostics. Measurements show a steep ion acceleration layer located 8 $\mathrm{cm}$ downstream the exit plane of the discharge channel, detached from any physical boundary of the plasma source. In this location, the ion velocity increases from $3 \mathrm{~km} / \mathrm{s}$ to $10 \mathrm{~km} / \mathrm{s}$ within a $1 \mathrm{~cm}$ axial region. The ion acceleration profile has been characterised under multiple testing conditions in order to identify the influence of the magnetic field intensity and topology on this peculiar ion acceleration layer.

\section{INTRODUCTION}

The Quad Confinement Thruster ${ }^{1}$ is a novel magnetized Direct Current plasma source developed for high specific impulse space propulsion applications. The QCT was originally proposed in 2010 at the Surrey Space Centre and underwent multiple development and industrialization programmes during the following years ${ }^{2}$, resulting in the first flight model launched in September 2018 on board the SSTL NovaSAR-1 satellite for an in-space technology demonstration. In a previous experimental characterisation $^{3}$, the QCT has shown a maximum specific impulse of $860 \mathrm{~s}$, a thrust of $3.2 \mathrm{mN}$ and an efficiency of $4.6 \%$ at the nominal anode power of $200 \mathrm{~W}$. A general schematic of the plasma source is provided in Fig.1. The device presents a square discharge channel with the anode of the system located at its closed end. The propellant enters the channel around the periphery of the anode plate and ionization is driven by electron-neutral collisions. An external hollow cathode discharge provides primary electrons for ionization and for neutralising the ejected ion beam. The magnetic field is characterized by four cusps located at the channel walls, generated by electromagnets. The resulting magnetic flux density is strongest at the walls and features a cancellation region in the center of the channel (see Fig.1). The cusps and magnetic null region extend throughout the length of the channel upward to the anode. The magnetic field constitutes a barrier to electron migration, enhancing the electron resident time within the discharge channel. Different combinations of current magnitudes can be supplied to the electromagnets to manipulate the magnetic field topology, changing the properties of the generated

\footnotetext{
a) Electronic mail: a.luccafabris@surrey.ac.uk

b) Presently at Lawrence Livermore National Laboratory, 7000 East Ave., Livermore, CA 94550, USA
}

plasma and potentially achieving an active thrust vector control without the use of a mechanical gimbal ${ }^{1}$. While the overall QCT propulsive performance has been extensively characterized in previous works ${ }^{3-6}$, this paper addresses the fundamental plasma physics in the thruster plume, with a particular focus on the ion acceleration mechanism.

The principle of operation of the QCT presents similarities with other plasma sources which exploit an $E \times B$ electron drift, such as Hall Effect Thrusters (HETs) ${ }^{7}$, cusped field thrusters ${ }^{8,9}$ and, in particular, the lineartype Hall thruster ${ }^{10}$. Typical Hall thruster plasma discharges present an axisymmetric geometry with an annular or a cylindrical discharge channel ${ }^{11}$ and a radial magnetic field generated by either a system of electromagnets or permanent magnets. In this layout, characterized by an axial electric field and a radial magnetic field, electrons are constrained in a closed $E \times B$ azimuthal drift ${ }^{12}$ generating a continuous Hall current loop. The $E \times B$ region is a volume typically confined close to the discharge channel exhaust plane and represents a zone of high ionization. Ions (unmagnetized for the magnetic field strength used in these devices) are subsequently accelerated by an electric field generated by the increased resistivity to axial electron motion through the channel imposed by the radial magnetic field. Electrons loop several times before migrating to the anode, yielding the condition $J_{e z} / J_{e H} \ll 1$, where $J_{e z}$ and $J_{e H}$ are the crossfield axial electron current and the Hall current respectively. Electron transport in Hall thrusters is a complex process which can not be described by the classical crossfield diffusion theory ${ }^{13,14}$; several anomalous transport mechanisms and models have been proposed in order to properly represent experimental observations.

Differently from the coaxial configuration, Hall plasma sources with a linear geometry are characterized by an open electron drift. In these devices the discharge channel presents a rectangular cross section with the mag- 
Figures/QCT_schematics_assembled.pdf

FIG. 1: Quad Confinement Thruster. (a) General schematic of the Quad Confinement Thruster. The discharge channel presents a square cross-section. Primary electrons produced by an external hollow cathode source (not shown) migrate toward the anode of the system ionizing the working gas via electron-neutral collisions. The neutral gas is injected at the periphery of the anode. (b) A magnetic field, characterized by cusps at the four lateral walls, impedes the electron axial transport creating an $E \times B$ electron drift with direction reversal in the four quadrants.

netic field aligned along the smaller dimension. As a consequence, the $E \times B$ drift develops along the perpendicular dimension and the electron path terminates at the channel wall ${ }^{10}$. The linear geometry HET, developed as an attempt to address the lack of efficient annular HETs in the low power range $(10-100 \mathrm{~W})$, presents man- ufacturing advantages because of its compact design and simpler magnetic circuit in comparison with the coaxial configuration. However, in the linear design the open electron drift may affect the discharge performance. The generation of an interrupted Hall current instead of a continuous Hall current can lead to a decrease in ion- 
Figures/Photograph2.pdf

FIG. 2: The discharge channel of the QCT presents peculiar light emission structures. The strongest light emission originates from two specific quadrants of the channel. The photograph shows a SSTL QCT

qualification model during testing at the European Space Agency Electric Propulsion Laboratory.

ization and limitations in establishing a potential gradient for ion acceleration. Similar considerations apply to the QCT discharge configuration. Indeed, the QCT is characterized by open $E \times B$ drifts, which point in opposite directions in contiguous quadrants due to the reversal of the magnetic field direction, as is illustrated in Fig.1. The open, alternating-direction electron drift induces a strong non-uniformity of the plasma discharge; visual observation of the device (Fig.2) shows a brighter plasma emission coming from the regions characterized by an outward $E \times B$ vector suggesting that the combination of electric and magnetic topologies drives electrons into this volume causing a higher number of excitationradiative relaxation events. A recent study of the QCT discharge using electron kinetic modeling ${ }^{15}$ has identified the $E \times B$ driven electron transport and the ambipolar diffusion as the physical mechanisms causing these peculiar visible light emission structures. Moreover, in the central magnetic-null region electrons may find a direct path to the anode leading to an increased axial electron current density in this portion of the channel crosssectional area, as experimentally observed in cusped field thrusters ${ }^{16}$. Once ions are produced in the channel, they are accelerated downstream by the electric field without being directly affected by the magnetic field because of their heavy mass. In the original QCT conceptual definition, the hypothetical mechanism for establishing this electric field was the impeded electron flow due to the transverse magnetic field barrier as in the HET case; however the experimental investigation presented in this paper shows a different physical framework. Moreover, previous studies have shown that plasma discharge properties are strongly influenced by the background residual pressure in the vacuum chamber ${ }^{6}$, suggesting a coupling between the physics of the plasma within the channel and that of the outer plume. By visual observation (Fig.3), the latter features a semi-spherical structure which extends about 6 - $8 \mathrm{~cm}$ from the thruster exit plane.

Figures/Photograph1.pdf

FIG. 3: Light emission structure from the plume of a QCT. The photograph was taken during testing of a SSTL QCT qualification model at the European Space Agency Electric Propulsion Laboratory.

Understanding the fundamental physics of the plasma discharge, particularly the ion acceleration mechanism, is a key step in evaluating and exploiting the thruster's propulsive capability and performance. Pursuant to this goal, we characterize the ion velocity field in the plasma ejected from a $200 \mathrm{~W}$ QCT laboratory model using a nonintrusive laser-induced fluorescence (LIF) diagnostic ${ }^{17,18}$. Time-averaged local measurements of most probable velocity vectors and corresponding velocity distribution functions are carried out for different magnetic configurations and anode voltages in several locations in the QCT plume providing an unprecedented picture of the ion flow-field. The conclusions achieved by this study have an important impact on the future optimization activities of the thruster since they represent a step-change in the understanding of the fundamental physics governing the plasma produced by the device. Two perpendicular velocity components have been characterized under different operating conditions and using different magnetic topologies generated by the flexible electromagnet 
arrangement. The steering of ion trajectories generated by the distortion of the magnetic field has also been investigated in order to verify the magnetic thrust vector control capability of the thruster.

\section{EXPERIMENTAL SETUP}

The QCT used for this study is a laboratory development model operating in the $200 \mathrm{~W}$ power range ${ }^{3}$. The discharge channel, made of boron nitride, presents a square cross section with a side of $35 \mathrm{~mm}$ and a length of $64 \mathrm{~mm}$. The tantalum anode has a square cross section as well, with a $25 \mathrm{~mm}$ side length. A commercial hollow cathode neutralizer has been used with its orifice located $3.2 \mathrm{~cm}$ downstream the channel exit plane and $9.4 \mathrm{~cm}$ outward from the centerline, pointing at about $60^{\circ}$. The magnetic field is generated by four electromagnets wrapped around blade-shaped soft iron rails which concentrate the field lines and create magnetic cusps at the midpoints of the four lateral walls (see schematic in Fig.1). The resulting field topology has a quadrupole configuration with the strongest magnitude at the walls and a cancellation point at the channel center. The magnitude of the magnetic field is roughly constant along the length of the channel and decreases toward the exit plane as shown in the experimental measurements and simulated profile reported in Fig.4a. Magnetic flux density maps in the near-field and mid-field plume regions are shown in Fig.4b. Since the QCT front plate is made of aluminum, the magnetic field leaks out from the iron rails and partially extends in the downstream region. Plots of the magnetic field topology obtained using a 3D magnetostatic simulation, details of which can be found $\mathrm{in}^{19}$, show strongly three dimensional field lines in this region. These field lines, as seen in Fig.5, exhibit bulging structures beyond the exit plane of the thruster that start to appear as soon as the field lines detach from the electromagnet blades and propagate further downstream into the plume region.

The test campaign is carried out at the Stanford Plasma Physics Laboratory. The QCT is mounted inside a $1.5 \mathrm{~m}$ diameter and $4 \mathrm{~m}$ long stainless steel cylindrical chamber, equipped with a two-stage cryogenic pumping system, reaching a base pressure of $4 \times 10^{-7}$ Torr without propellant flow and maintaining $3 \times 10^{-5}$ Torr (uncorrected for xenon) while operating the thruster. The background pressure might play a critical role in the experimental characterisation of the QCT. A prior interlaboratory characterisation of the $\mathrm{QCT}^{6}$, performed at the Surrey Space Centre and European Space Agency Electric Propulsion laboratories, demonstrated a significant change of the QCT voltage-current characteristics when the background pressure was varied in the 3.8 $9 \times 10^{-5}$ Torr range using an additional mass flow input. Variations of the measured thrust were also observed. Extensive literature describes the effects of the background pressure on Hall Effect Thrusters testing re- vealing an influence on the performance, discharge channel physics and plume characteristics ${ }^{20-22}$. In particular, relevant to the results presented in this article, a recent study $^{23}$ has analysed the effect of the background pressure in the range $1.7-8 \times 10^{-5}$ Torr on xenon ion velocity distribution functions in a SPT-100 thruster plume characterised through laser-induced fluorescence velocimetry. The analysis shows the background pressure influences the ionization and acceleration zones, with an observed upstream shift of the axial ion acceleration front when the pressure is increased; nevertheless, the ultimate ion velocity after acceleration appears unaffected.

The local ion velocity components in the QCT plume are measured by applying a non-intrusive laser-induced fluorescence technique, extensively described in previous literature $^{18,24,25}$. A schematic of the LIF system experimental setup is illustrated in Fig.6. The ion velocity is determined by the Doppler shift of the LIF spectrum of the QCT plume plasma relative to the optogalvanic spectrum of a stationary plasma in a hollow cathode lamp. The Xe II $5 d[4]_{7 / 2}-6 p[3]_{5 / 2}$ transition at 834.953 $\mathrm{nm}$ (vacuum wavelength) is optically pumped by a laser beam generated by a New Focus TA-7600 semiconductor tapered amplifier seeded by a $20-\mathrm{mW}$ tunable externalcavity diode laser (New Focus, Model TLB-6817-P). The fluorescence at $542.1 \mathrm{~nm}$ (vacuum), resulting from the radiative decay to the $6 s[2]_{3 / 2}$ state, is collected by a $100 \mathrm{~mm}$ diameter, $200 \mathrm{~mm}$ focal length lens within the chamber, directed outside through a set of mirrors and focused onto a Hamamatsu 1P21 photomultiplier tube (PMT). A $10 \mathrm{~nm}$ bandpass filter, centered at $540 \mathrm{~nm}$, and an optical short-pass filter are mounted in front of the PMT to reject photons originating from transitions other than the targeted one. An additional mechanical iris is placed in front of the PMT in order to reduce the light collection volume. The intersection between the collection optical path and the probe laser beam determines the measurement volume with typical size of $\sim 1$ $\mathrm{mm}^{326,27}$. A translation stage system allows movement of the thruster along two directions, enabling spatiallyresolved measurements.

The laser wavelength is scanned throughout a $30 \mathrm{GHz}$ range, encompassing the Doppler-shifted ion fluorescence spectrum resulting from the QCT plasma and the stationary reference spectrum resulting from the plasma generated in the hollow cathode lamp. The detuning process is monitored by a Fabry-Perot interferometer whose interference fringes are spaced by $1.5 \mathrm{GHz}$. An additional wavemeter provides a visual wavelength reading while setting the laser operating parameters. The Xe I transition probed in the hollow cathode lamp is centered at $834.973 \mathrm{~nm}$ (vacuum), 9.03 GHz from the line center of the stationary ion transition. A previous experimental analysis using an independent stationary plasma source determined a $360 \mathrm{~m} / \mathrm{s}$ velocity accuracy for a similar LIF setup $^{25}$ with the same critical optical components and wavelength reconstruction method.

Before entering into the vacuum chamber through a 
Figures/magnetic_flux_density.pdf

FIG. 4: Simulated magnetic flux density for the QCT. The simulations are obtained using a 3D model of the thruster's magnetic field built with EMWork's EMS software. (a) Axial profile of the magnetic flux density at the center of a side wall of the discharge channel (comparison between measured and simulated values). (b) Magnetic flux density maps on the $\mathrm{Y}=0$ plane; bottom map: near field plume (region between $\mathrm{Z}=0$ and $\mathrm{Z}=40 \mathrm{~mm}$ ); top map: plume region between $\mathrm{Z}=60 \mathrm{~mm}$ and $\mathrm{Z}=100 \mathrm{~mm}$.

side window, the laser beam is split into two beams which are directed to the thruster plume from perpendicular directions using a set of mirrors. This arrangement allows for the simultaneous measurement of two perpendicular velocity components (Fig.7). The two beams are modulated at different frequencies using a mechanical chopper enabling the application of homodyne detection through lockin amplifiers to discriminate fluorescence originating from one or the other beam and to filter-out the noise content from the PMT signal. The ion velocity measurements are performed on a symmetry plane of the thruster and the resulting ion velocity maps are illus- 
Figures/magnetic_field_topology.pdf

FIG. 5: Simulated magnetic field topology of the QCT, using a 3D model of the thruster's magnetic field built with EMWork's EMS software. (a) Bulging 3D structures of the magnetic field lines detaching from the electromagnet blades and extending into the plume region. (b) Top view of the magnetic field lines launched at $\mathrm{Z}=80 \mathrm{~mm}$. (c)

Front view of the magnetic field lines.

trated and discussed in the next section. Measurements are performed operating the thruster under a broad range of conditions; in particular, the electromagnet currents have been widely varied to study the effect of the magnetic field distortion on the ion flow field.

\section{RESULTS AND DISCUSSION}

The plasma discharge operates with a xenon flow of $0.8 \mathrm{mg} / \mathrm{s}$ through the anode and an argon flow of 0.12 $\mathrm{mg} / \mathrm{s}$ through the external hollow cathode discharge. The use of argon allows to exclusively probe ions originating from the main discharge channel and avoids the detection 
Figures/LIF_lay_out.pdf

FIG. 6: Schematic of the LIF system experimental setup, optical components and equipment.

of ion populations originating from the hollow cathode plasma, which can introduce misleading interpretations of the results.

The following set of data is obtained for the standard magnetic field configuration (i.e. using a current of $1 \mathrm{~A}$ through all the electromagnet windings, generating the magnetic field shown in Figs.1,4 and 5) and a fixed anode voltage of $130 \mathrm{~V}$. The anode current experiences a long-term drift (over timescales of 20 - $30 \mathrm{~min}$ ) within the range $1.5-1.8 \mathrm{~A}$ during the several hours of continuous thruster operation. The short-term dynamic behavior of the discharge current is monitored through a shunt resistor and high-voltage common mode differential amplifier; the current presents a quiescent profile without any relevant coherent oscillation within the $0-120 \mathrm{kHz}$ sensitivity range of the measurement system. No significant short-term change of the discharge current is observed while moving the thruster from one location to another using the translation stages for spatially-resolved measurements.

Spatial maps of most probable ion velocity components are presented in Fig.8 using the same reference frame illustrated in Figs. 1 and 5 and depicted again Fig.7. The origin of the frame is located at the exit plane of the thruster on the discharge channel centerline. The $\mathrm{X}$ and $\mathrm{Y}$ axis are aligned with the diagonals of the square discharge channel and the $\mathrm{Z}$ axis is aligned with the channel axis of symmetry. Measurements are taken on the XZ plane. The axial velocity map highlights that a steep increase in ion velocity is taking place at about $8 \mathrm{~cm}$ downstream of the exit with an observed increase from 3 $\mathrm{km} / \mathrm{s}$ to $10 \mathrm{~km} / \mathrm{s}$ (on the centerline) within a region of 1
Figures/LIF_layout_inner.pdf

FIG. 7: Two laser beams modulated at different frequencies $f_{1}$ and $f_{2}$ are used for probing the axial and transverse velocity components in the QCT plume. The

fluorescence photons are collected by a lens and redirected outside the chamber by means of a set of mirrors. The measurement volume is given by the intersection of the probing laser beams and the collection optical path. The figure also shows the $X Z$ reference frame used in the presentation of the results.

cm thickness or less. This layer is located in "free space", i.e. in a region detached from the exit plane and physical boundaries, characterized by weak magnetic field intensity and lower neutral density in comparison with the discharge channel. The location of the acceleration front is atypical and differs from the one observed in other types of open-channel thrusters, such as Hall Effect Thusters ${ }^{17}$ and Cusped Field Thrusters ${ }^{24}$. Indeed, in these latter devices, ion acceleration takes place around the exit plane: in some cases the front extends just few centimeters beyond the exit ${ }^{17}$, in other cases it is even located inside the channel $^{27}$. The observation of the velocity vectors and angles on the centreline of the thruster, shown in Fig.8d and Fig. $8 \mathrm{c}$ respectively, reveals a $\sim 5$ deg angular offset of the velocity vectors toward the positive $\mathrm{X}$ axis, suggesting the presence of slight asymmetries in the thruster discharge or a small misalignment of the thruster with respect to the optical components of the experimental setup. The location of the acceleration front is highlighted further in Fig.9, representing the axial evolution of the axial Ion Velocity Distribution Function along a line located at a transverse coordinate $X=-30 \mathrm{~mm}$. The profile features a sharp acceleration at an axial coordinate of $75-80 \mathrm{~mm}$ from the exit plane with a most probable ion velocity increase from $1 \mathrm{~km} / \mathrm{s}$ to $8 \mathrm{~km} / \mathrm{s}$. 
Figures/M1_axial_vel.pdf

Figures/M1_angle.pdf
Figures/M1_transv_vel.pdf

Figures/M1_vel_vectors.pdf

FIG. 8: Ion velocity measurements in case of nominal magnetic field. (a) Measured axial velocity map. (b) Measured transverse velocity map. (c) Computed velocity angle. (d) Computed velocity vectors. Triangle markers indicate the location of the LIF measurements. In subplot (b), negative and positive values are shown with the same colour scale to represent the transverse velocity magnitude; however, the transverse velocity is negative for $X<0$, and positive for $X>0$, i.e. this velocity component is always pointing outward from the centreline as inferred from the velocity vectors shown in subplot $(d)$.

The peculiar location of the acceleration front in the $\mathrm{QCT}$ is due to factors determining the underlying plasma physics in the plume and in the discharge channel. A full description of the physical processes would require a comprehensive investigation comprising multiple probes, however the known physical features of the device al- low the formulation of a hypothesis. The nature of the acceleration is electrostatic and ion acceleration is triggered by a potential drop, the position of which matches that of the observed acceleration front. Potential drops in narrow spatial regions detached from plasma boundaries have been observed in other laboratory plasmas ${ }^{28}$ 
Figures/IVDF_Vz_x_30.pdf

FIG. 9: Axial profile of the axial LIF signal (representing the axial Ion Velocity Distribution Function) in the plasma plume along a line located at $X=-30 \mathrm{~mm}$. LIF scans have been weighted by the integrated scan intensity and normalised to the overall maximum.

and are usually defined as single or double layers ${ }^{29}$ depending on their characteristics. As an example, single and double layers have been reported in multiple plasma devices with electron drift current ${ }^{30,31}$ or in magnetized expanding plasmas ${ }^{32,33}$. The location of the acceleration layer observed in the QCT plume suggests a similar structure. Electrons flowing from the hollow cathode remain attached to the magnetic field lines leaking from the iron rails of the thruster (see Fig.5) and move throughout a semi-spherical shell with a certain thickness and certain radius depending on the relative position of the hollow cathode orifice and magnetic field topology. This electron rich shell creates a local breakdown of quasineutrality, creating a potential jump bounded between an ion-rich region (upstream towards the thruster) and an electron-rich region (downstream). Particle kinetic phase-spaces are characterized by trapped low-energy electrons on the high potential side, a population of drifting electrons accelerating from the low-potential side to the high-potential side of the structure migrating from the electron-rich shell towards the discharge channel, and an accelerated beam of ions gaining energy from the potential jump. The shape of the potential front influences the direction of ion acceleration occurring in the direction of the potential gradient. As a result of the spatial distribution in the potential, the velocity vectors are aligned to the z-direction at the centre of the plume and their angle progressively increases moving outward and reaches a value of about $65-70^{\circ}$ at the periphery (see Fig.8d). Visual observation of the QCT plume reveals plasma emission is preferentially originating from a semi-spherical region in front of the thruster with a radius comparable to that of the identified acceleration front, suggesting a correlation between electrostatic topology and visual shape of the plume (Fig.3).

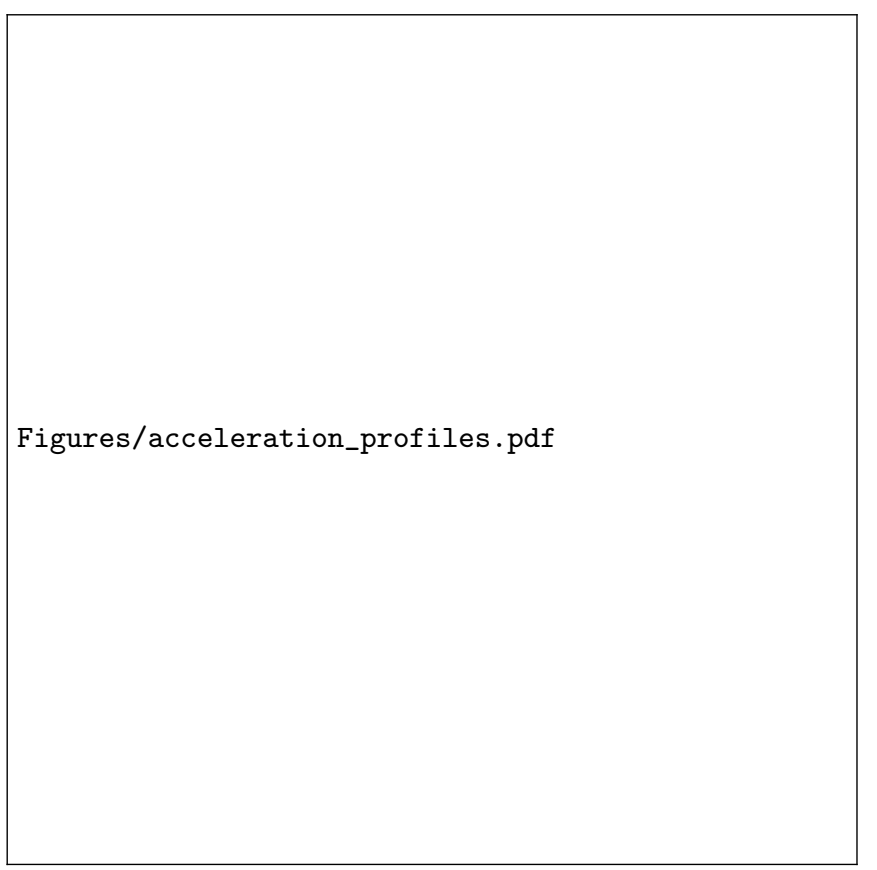

FIG. 10: Ion acceleration profiles under different testing conditions. The black circles report the most probable ion velocity at different axial locations from the exit plane for nominal operating conditions. The green triangles represent the case with reduced magnetic field (half of the nominal magnitude). The yellow squares identify the acceleration front at reduced power operation (100 W power with $100 \mathrm{~V}$ anode voltage).

The red triangle shows the weaker ultimate velocity of ions when the plasma discharge operates without magnetic field.

Fig.10 reports the most probable ion axial velocity in different locations in the plume along the thruster centerline for different testing conditions. The ion velocity profile obtained for the nominal magnetic field case (black line in the figure) highlights again the velocity increase from $3 \mathrm{~km} / \mathrm{s}$ to $10 \mathrm{~km} / \mathrm{s}$ at about $8 \mathrm{~cm}$ from the exit plane. Conversely, the measured ion velocity for the unmagnetized (i.e., null magnetic field) discharge case (red triangle in the figure) presents a value of only 4 $\mathrm{km} / \mathrm{s}$ at $11 \mathrm{~cm}$ from the exit plane, equivalent to a potential drop limited to $10-15 \mathrm{~V}$. The comparison between measurements obtained applying the nominal magnetic field and that acquired for the unmagnetized discharge mode demonstrates that strong ion acceleration takes place only in the magnetized case. This highlights the impact of the magnetic field on the discharge physics, 
Figures/nominal_B_frontview.pdf
Figures/asymmetric_B_frontview.pdf

FIG. 11: Comparison of magnetic flux density and magnetic field lines in the QCT discharge channel between case (a) with nominal magnetic field (all electromagnets are powered) and case (b) with asymmetric magnetic field (only electromagnets $\mathrm{C}$ and $\mathrm{D}$ are powered).

in particular on the electrostatic potential distribution determining ion acceleration. A decrease of the anode power (case with $100 \mathrm{~V}$ anode voltage and $1 \mathrm{~A}$ anode current) causes a weakening of the acceleration, due to the reduction of the potential drop strength resulting from the decrease of the anode voltage level. Finally, a reduction of the magnetic field strength to half of the nominal value does not produce any significant change to the velocity increase experienced by ions. The location of the acceleration layer remains approximately fixed, at about $80-85 \mathrm{~mm}$ downstream of the exit plane, for all the tested conditions with magnetic field. This latter observation reveals that the acceleration front is connected to the system physical geometry and magnetic topology.

The decoupling of ionization and acceleration regions and the generation of cross-field wall-less plasmas have been the subject of much research within the space propulsion community, aimed at mitigating wall-losses and reducing the erosion of the ceramic channel ${ }^{34-37}$ thus extending the operational lifetime. These studies have demonstrated the feasibility of such discharge with ionization and acceleration regions located in free space outside of a physical channel. A major drawback of these devices is the high divergence of the ion velocity that causes substantial radial losses. For the same reason, the QCT generally presents a lower performance in comparison with standard Hall Effect Thrusters.

The magnetic field was altered by switching off two electromagnets. For the LIF measurements reported in Fig.12, only electromagnets $\mathrm{C}$ and $\mathrm{D}$ are powered (the four electromagnets are labelled using the letters A,B,C,D according to the lay-out shown in Fig.1.). The resulting asymmetric magnetic field is illustrated in Fig.11, along with the nominal field shown for comparison. The figure highlights the drastic decrease of the magnetic field in the two quadrants of the discharge channel on the side of the two unpowered electromagnets as well as a strong re-arrangement of the magnetic field lines with a strong cusp structure in the opposite quadrants. The discharge is therefore characterised by a higher magnetic field half and a lower magnetic field one. The distortion of the magnetic topology generates changes in both the ion velocity field and the metastable (probed) ion density. A non-uniform distribution of metastable ions in the plume is observed, with higher LIF signal observed on the $X<0$ side, as illustrated in Fig.13. The acceleration front is still located at about $8 \mathrm{~cm}$ from the exit plane with an axial velocity increase from $3 \mathrm{~km} / \mathrm{s}$ to $10 \mathrm{~km} / \mathrm{s}$ taking place in a $1 \mathrm{~cm}$ region (see Fig.12a) as in the case of the nominal magnetic configuration. However, a higher transverse velocity component is observed on the $X>0$ side with a consequent change in the velocity angle (see Fig.12b and Fig. 12c, respectively). In this specific case we can observe a transverse velocity delta of about $2 \mathrm{~km} / \mathrm{s}$ between the two sides of the midplane $(\mathrm{X}=0)$. The asymmetric magnetic field induces a non-uniform electrostatic potential distribution within the plasma, with a consequent effect on ion dynamics. These results demonstrate the impact of the magnetic field configuration on the ion velocity vector, providing 
Figures/M2_axial_vel.pdf

Figures/M2_angle.pdf
Figures/M2_transv_vel.pdf

Figures/M2_vel_vectors.pdf

FIG. 12: Ion velocity measurements in case of distorted magnetic field. Only two electromagnets are powered (Magnet C and D). (a) Measured axial velocity map. (b) Measured transverse velocity map. (c) Computed velocity angle. (d) Computed velocity vectors. Triangle markers indicate the location of the LIF measurements. In subplot

(b), negative and positive values are shown with the same colour scale to represent the transverse velocity

magnitude; however, the transverse velocity is negative for $X<0$, and positive for $X>0$, i.e. this velocity component is always pointing outward from the centreline as inferred from the velocity vectors shown in subplot (d).

a preliminary assessment on the potential for thrust vectoring. However, the latter capability needs to be corroborated by either complementary measurements of the ion flux or multi-directional direct thrust measurements. As an example, a previous study on magnetic steering of the ion beam of a stationary plasma thruster combined probe measurements with thrust balance data for computing the thrust-vector deviation angle ${ }^{38}$ and successfully demonstrated a steering of 5 deg of the trust vector. Differently from the QCT, in which the main magnetic system of the thruster is used, this previous investigation included an external magnetic system to directly deviate 
Figures/M2_axial_LIF_intensity.pdf

FIG. 13: Measured axial LIF signal intensity in case of distorted magnetic field with only two electromagnets $(\mathrm{C}$ and $\mathrm{D})$ powered. Triangle markers indicate the location of the LIF measurements.

the trajectories of xenon ions in the plasma jet. Finally, the impact on the QCT global performance metrics and erosion patterns of the channel wall caused by an asymmetrical field also needs to be assessed for drawing a final conclusion.

\section{CONCLUSIONS}

Laser-Induced Fluorescence ion velocimetry mapping on a Quad Confinement Thruster (probing the Xe II $5 d[4]_{7 / 2}-6 p[3]_{5 / 2}$ metastable ion transition at 834.953 $\mathrm{nm}$ ) has revealed the presence of a free-space ion acceleration layer that is detached from the main physical boundaries of the plasma discharge. The ion velocity field exhibits an increase in axial velocity from $3 \mathrm{~km} / \mathrm{s}$ to $10 \mathrm{~km} / \mathrm{s}$ within a spatial region of $1 \mathrm{~cm}$ or less approximately $8 \mathrm{~cm}$ downstream of the thruster exit. Measurements have demonstrated the location of this acceleration front does not change when the magnetic field intensity is reduced to half of the nominal value; however, a strong reduction of the ultimate ion velocity is observed for the unmagnetized case (null magnetic field), suggesting the occurrence of the strong acceleration layer is linked to the presence of the magnetic field. We attribute the cause of this detached acceleration region to the presence of an electrostatic potential structure similar to a double-layer. Experiments have been performed for different magnetic field topologies in order to better understand the effect of varying the B-field on the ion velocities. The mag- netic field topology is found to influence the distribution of the plasma properties resulting in a different electrostatic potential structure responsible for ion acceleration. Intentional asymmetries about the mid-plane generated in the magnetic field result in corresponding asymmetries in the laser-induced fluorescence signal intensity as well as in the transverse ion velocity. This study represents a key step-forward in understanding the characteristics of the ion acceleration process in the Quad Confinement Thruster, a novel plasma propulsion technology that has been recently launched on board the NovaSAR-1 satellite for an in-space technology demonstration. The discovery of the peculiar presence of an ion acceleration layer detached from the physical boundaries of the discharge channel represents an interesting plasma physics problem that deserves a dedicated future investigation using an array of probes for revealing its fundamental physics.

\section{ACKNOWLEDGEMENTS}

This research was supported in part by the U.S. Air Force Office of Scientific Research under grant FA955014-1-0017 with Dr. Mitat Birkan as Program Manager. The development of the QCT at the University of Surrey was supported by Airbus DS.

\section{DATA AVAILABILITY STATEMENT}

The datasets analyzed for this study shall be available upon request.

\section{REFERENCES}

${ }^{1}$ A. Knoll, B. Melly, and V. Lappas, "The quad confinement thruster-preliminary performance characterization and thrust vector control," in Proc. 32nd Int. Electr. Propuls. Conf., IEPC2011-099 (2011).

${ }^{2}$ O. Lane and A. Knoll, "Quad confinement thrusterindustrialisation \& flight integration," in Proc. 34 nd Int. Electr. Propuls. Conf., IEPC-2015-036 (2015).

${ }^{3}$ A. Knoll, T. Harle, V. Lappas, and M. Pollard, "Experimental performance characterization of a two-hundred-watt quad confinement thruster," Journal of Propulsion and Power 30, 14451449 (2014)

${ }^{4}$ T. Harle, A. Knoll, V. Lappas, and P. Bianco, "Performance measurements of a high powered quad confinement thruster," in 33rd International Electric Propulsion Conference, IEPC 2013283 (Washington, D.C., USA, 2013).

${ }^{5}$ A. Knoll, D. Lamprou, V. Lappas, M. Pollard, and P. Bianco, "Thrust balance characterization of a $200 \mathrm{w}$ quad confinement thruster for high thrust regimes," IEEE Transactions on Plasma Science 43, 185-189 (2015).

${ }^{6}$ A. Lucca Fabris, A. Knoll, K. Dannenmayer, T. Schönherr, T. Potterton, and P. Bianco, "Vacuum facility effects on quad confinement thruster testing," in 35th International Electric Propulsion Conference, IEPC-2017-250 (2017)

${ }^{7}$ M. A. Cappelli, "The Hall effect and rocket flight," Physics Today 62, 76 (2009) 
${ }^{8}$ N. Koch, M. Schirra, S. Weis, A. Lazurenko, B. van Reijen, J. Haderspeck, A. Genovese, P. Holtmann, R. Schneider, K. Matyash, et al., "The hempt concept-a survey on theoretical considerations and experimental evidences," in Proc. 32nd Int. Electr. Propuls. Conf., IEPC-2011-236 (2011).

${ }^{9}$ N. MacDonald, C. Young, M. Cappelli, and W. Hargus Jr, "Ion velocity and plasma potential measurements of a cylindrical cusped field thruster," Journal of Applied Physics 111, 093303 (2012).

${ }^{10}$ D. Schmidt, N. Meezan, W. Hargus Jr, and M. Cappelli, "A lowpower, linear-geometry hall plasma source with an open electrondrift," Plasma Sources Science and Technology 9, 68 (2000).

${ }^{11}$ Y. Raitses and N. Fisch, "Parametric investigations of a nonconventional hall thruster," Physics of Plasmas 8, 2579-2586 (2001).

${ }^{12}$ V. Zhurin, H. Kaufman, and R. Robinson, "Physics of closed drift thrusters," Plasma Sources Science and Technology 8, R1 (1999).

${ }^{13}$ M. K. Scharfe, N. Gascon, M. A. Cappelli, and E. Fernandez, "Comparison of hybrid hall thruster model to experimental measurements," Physics of Plasmas 13, 083505 (2006).

${ }^{14} \mathrm{~F}$. Taccogna, S. Longo, M. Capitelli, and R. Schneider, "Anomalous transport induced by sheath instability in hall effect thrusters," Applied Physics Letters 94, 251502 (2009).

${ }^{15}$ R. D. Williams, A. Lucca Fabris, and A. Knoll, "Insight into the plasma structure of the quad confinement thruster using electron kinetic modelling," Acta Astronautica 173, 111-118 (2020).

${ }^{16}$ H. Wu, H. Liu, Y. Meng, J. Zhang, S. Yang, P. Hu, P. Chen, and D. Yu, "Anode current density distribution in a cusped field thruster," Physics of Plasmas 22, 124504 (2015).

${ }^{17}$ R. J. Cedolin, W. A. Hargus, Jr., P. V. Storm, R. K. Hanson, and M. A. Cappelli, "Laser-induced fluorescence study of a xenon Hall thruster," Applied Physics B 65, 459-469 (1997).

${ }^{18} \mathrm{~S}$. Mazouffre, "Laser-induced fluorescence diagnostics of the cross-field discharge of Hall thrusters," Plasma Sources Science and Technology 22, 013001 (2013).

${ }^{19}$ E. Rosati Azevedo, V. G. Tirila, A. Schwertheim, and A. Knoll, "Magnetic field enhancement of the quad confinement thruster (qct): design and early development of the qct phoenix," in Proceedings of the 7th Space Propulsion Conference (2021).

${ }^{20}$ T. Randolph, V. Kim, H. Kaufman, K. Kozubsky, V. Zhurin, and M. Day, "Facility effects on stationary plasma thruster testing," in 23rd International Electric Propulsion Conference, 844 (1993) pp. 13-16.

${ }^{21}$ M. L. Walker, A. L. Victor, R. R. Hofer, and A. D. Gallimore, "Effect of backpressure on ion current density measurements in hall thruster plumes," Journal of Propulsion and Power 21, 408415 (2005)

${ }^{22}$ K. Diamant, R. Spektor, E. Beiting, J. Young, and T. Curtiss, "The effects of background pressure on hall thruster operation," in 48th AIAA/ASME/SAE/ASEE Joint Propulsion Conference ES Exhibit (2012) p. 3735.
${ }^{23}$ N. MacDonald-Tenenbaum, Q. Pratt, M. Nakles, N. Pilgram, M. Holmes, and W. Hargus Jr, "Background pressure effects on ion velocity distributions in an spt-100 hall thruster," Journal of Propulsion and Power 35, 403-412 (2019).

${ }^{24}$ A. Lucca Fabris, C. V. Young, and M. A. Cappelli, "Timeresolved laser-induced fluorescence measurement of ion and neutral dynamics in a hall thruster during ionization oscillations," Journal of Applied Physics 118, 233301 (2015).

${ }^{25}$ A. Lucca Fabris, C. V. Young, M. Manente, D. Pavarin, and M. A. Cappelli, "Ion velocimetry measurements and particle-incell simulation of a cylindrical cusped plasma accelerator," IEEE transactions on plasma science 43, 54-63 (2015).

${ }^{26}$ A. Lucca Fabris, C. V. Young, and M. A. Cappelli, "Timeresolved laser-induced fluorescence measurement of ion and neutral dynamics in a Hall thruster during ionization oscillations," Journal of Applied Physics 118, 233301 (2015).

${ }^{27}$ C. Young, A. Lucca Fabris, N. MacDonald-Tenenbaum, W. Hargus, and M. Cappelli, "Time-resolved laser-induced fluorescence diagnostics for electric propulsion and their application to breathing mode dynamics," Plasma Sources Science and Technology 27, 094004 (2018).

${ }^{28}$ N. Hershkowitz, "Review of recent laboratory double layer experiments," Space science reviews 41, 351-391 (1985).

${ }^{29} \mathrm{C}$. Charles, "A review of recent laboratory double layer experiments," Plasma Sources Science and Technology 16, R1 (2007).

${ }^{30}$ P. Leung, A. Wong, and B. Quon, "Formation of double layers," The Physics of Fluids 23, 992-1004 (1980).

${ }^{31}$ A. Sekar and Y. Saxena, "Observations of strong double layers in a double plasma device," Plasma physics and controlled fusion 27, 673 (1985)

${ }^{32} \mathrm{D}$. Andersson, "Double layer formation in a magnetised laboratory plasma," Journal of Physics D: Applied Physics 14, 1403 (1981).

${ }^{33}$ F. F. Chen, "Physical mechanism of current-free double layers," Physics of plasmas 13, 034502 (2006).

${ }^{34}$ C. Thomas, E. Sommier, N. Gascon, and M. Cappelli, "The magnetic interference hall accelerator," in 42nd AIAA/ASME/SAE/ASEE Joint Propulsion Conference \& Exhibit (2006) p. 4993.

${ }^{35}$ S. Mazouffre, S. Tsikata, and J. Vaudolon, "Development and experimental characterization of a wall-less Hall thruster," Journal of Applied Physics 116, 243302 (2014).

${ }^{36}$ J. Vaudolon, S. Mazouffre, C. Hénaux, D. Harribey, and A. Rossi, "Optimization of a wall-less hall thruster," Applied Physics Letters 107, 174103 (2015).

${ }^{37}$ B. Karadag, S. Cho, and I. Funaki, "Thrust performance, propellant ionization, and thruster erosion of an external discharge plasma thruster," Journal of Applied Physics 123, 153302 (2018).

${ }^{38}$ A. Bugrova, G. Bugrov, A. Bishaev, A. Desyatskov, M. Kozintseva, A. Lipatov, V. Kharchevnikov, and P. Smirnov, "Experimental investigation of thrust-vector deviation in a plasma thruster," Technical physics letters 40, 161-163 (2014). 


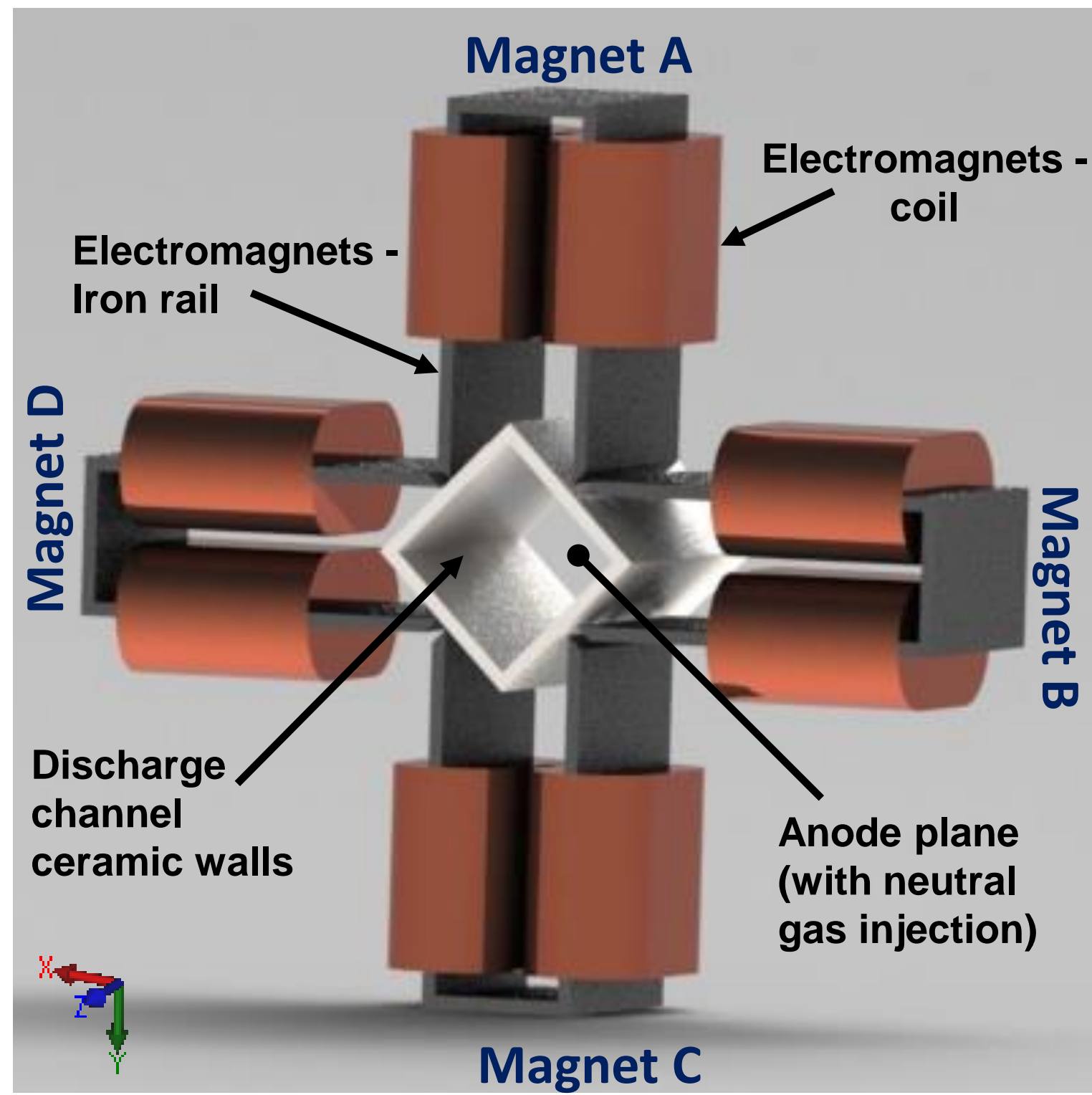

(a)

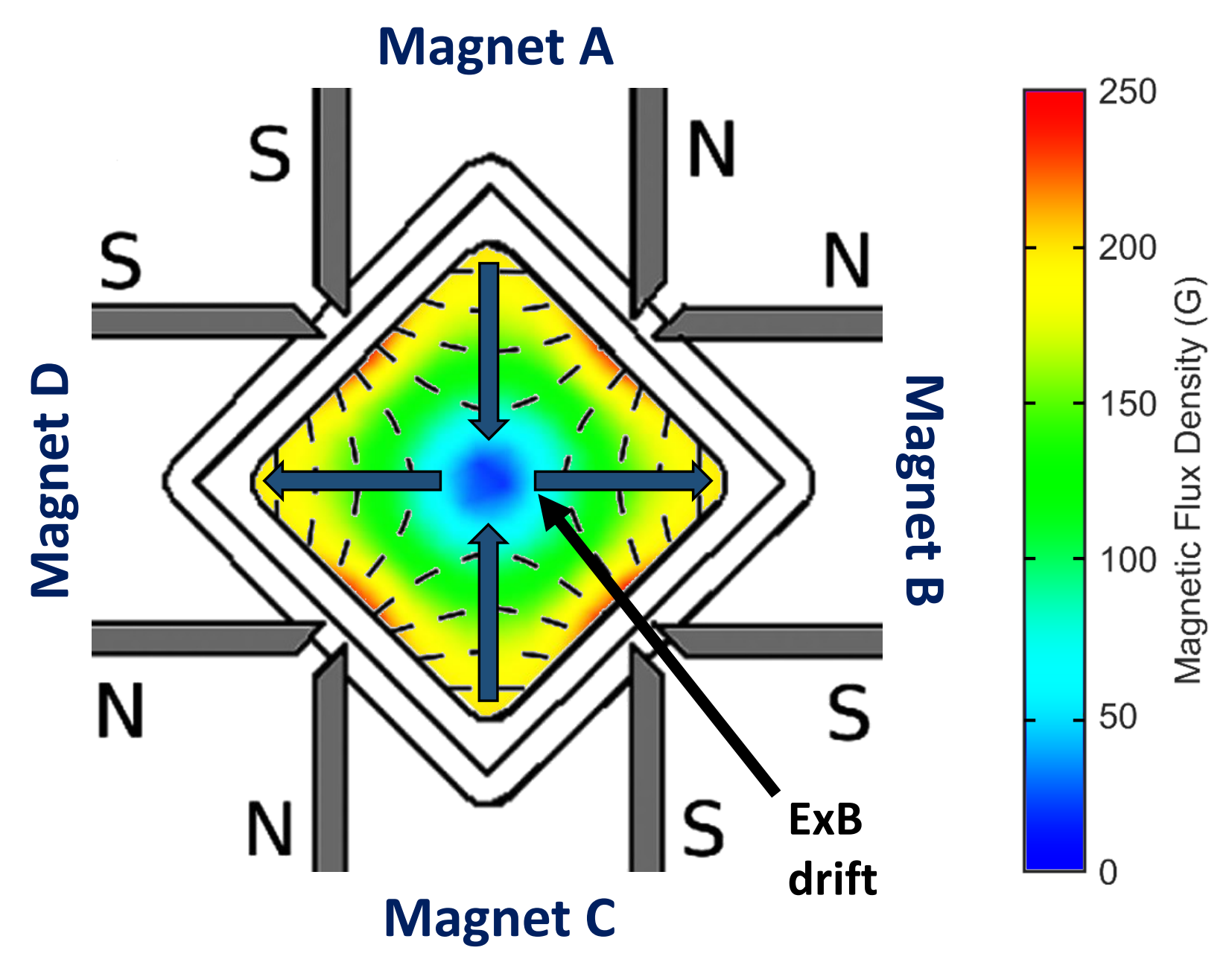

(b) 


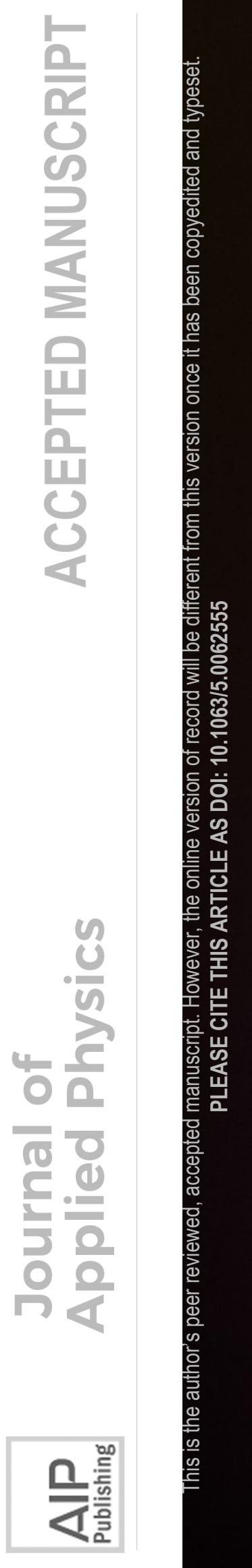



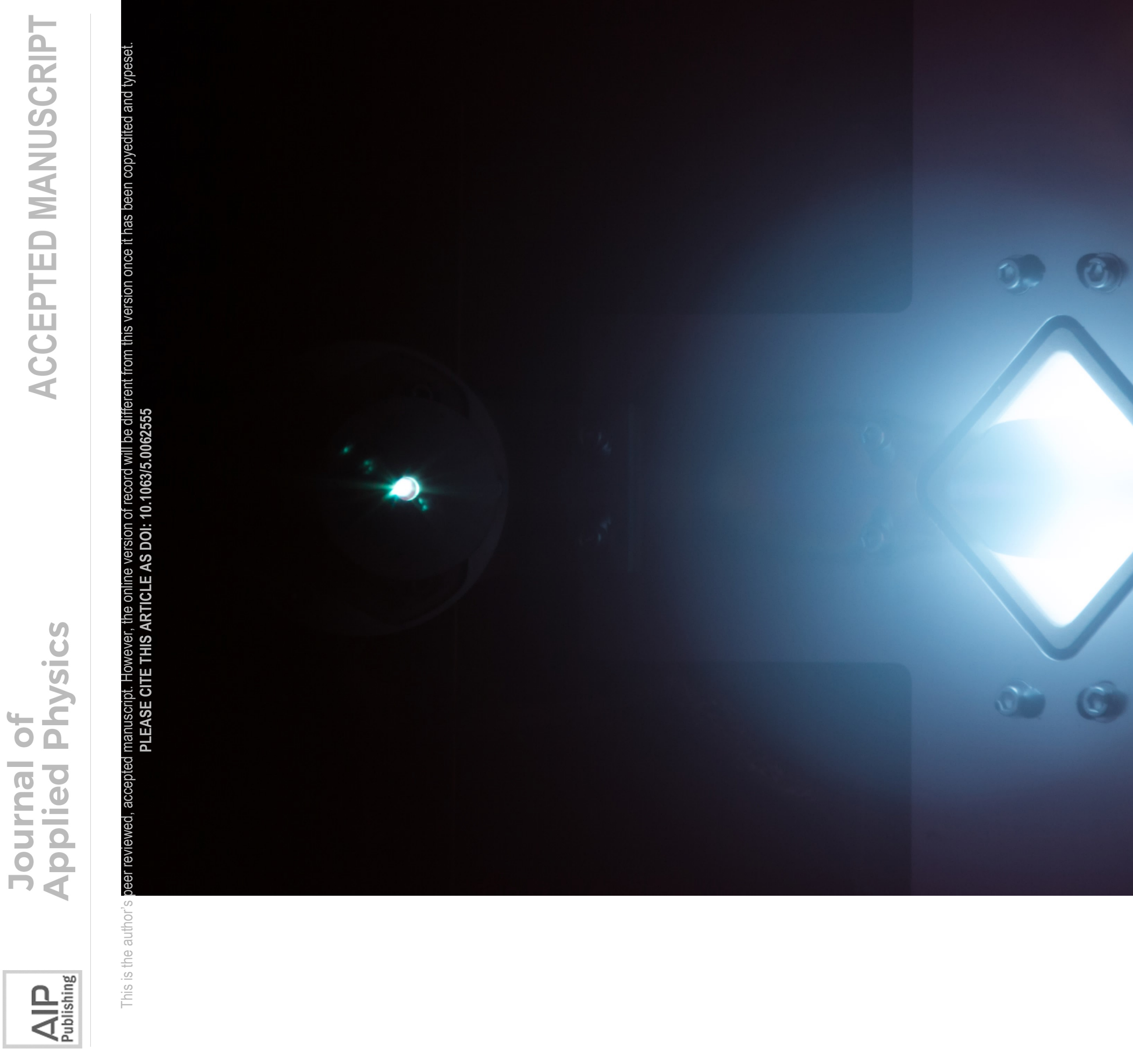


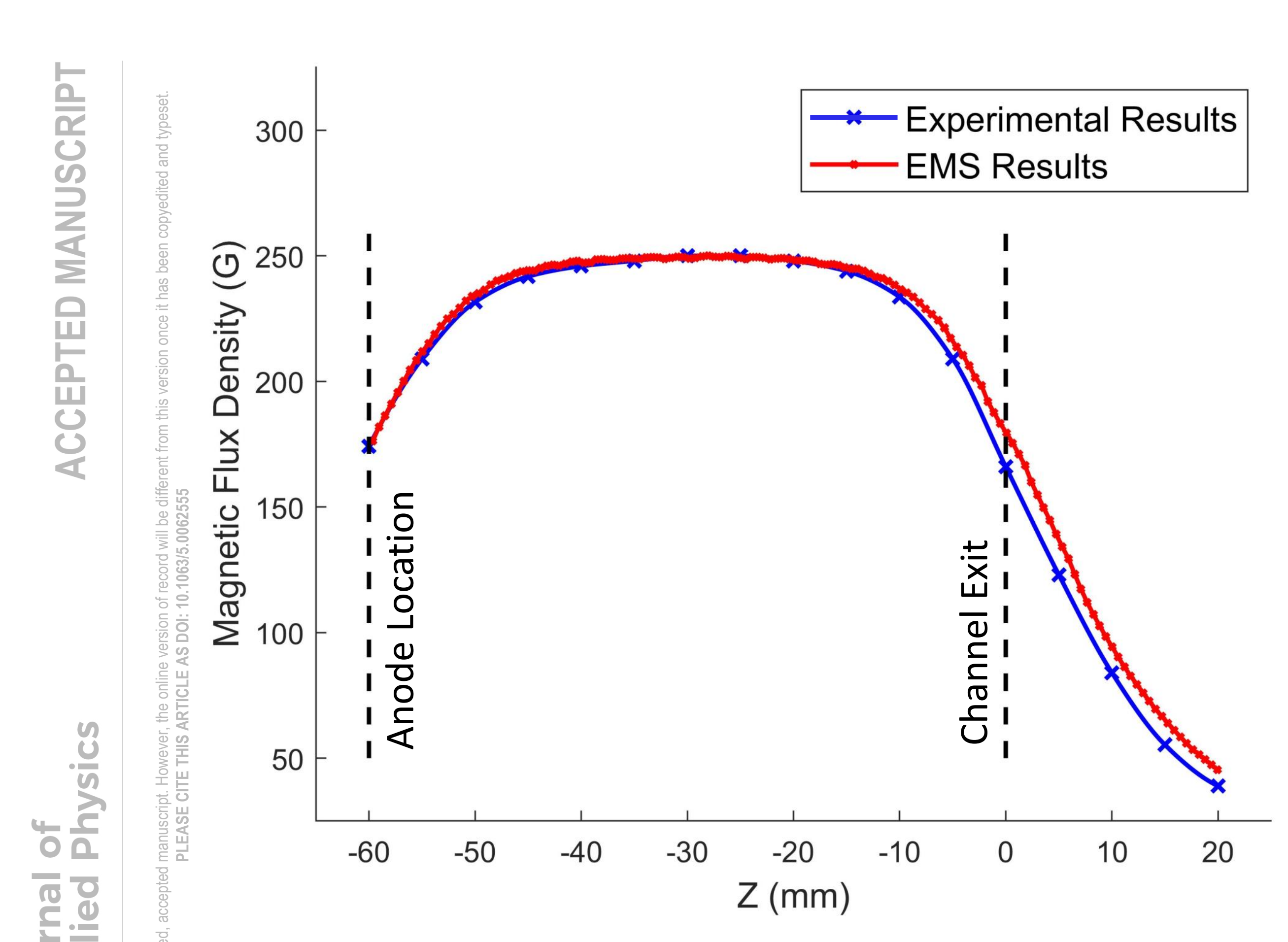

(a)
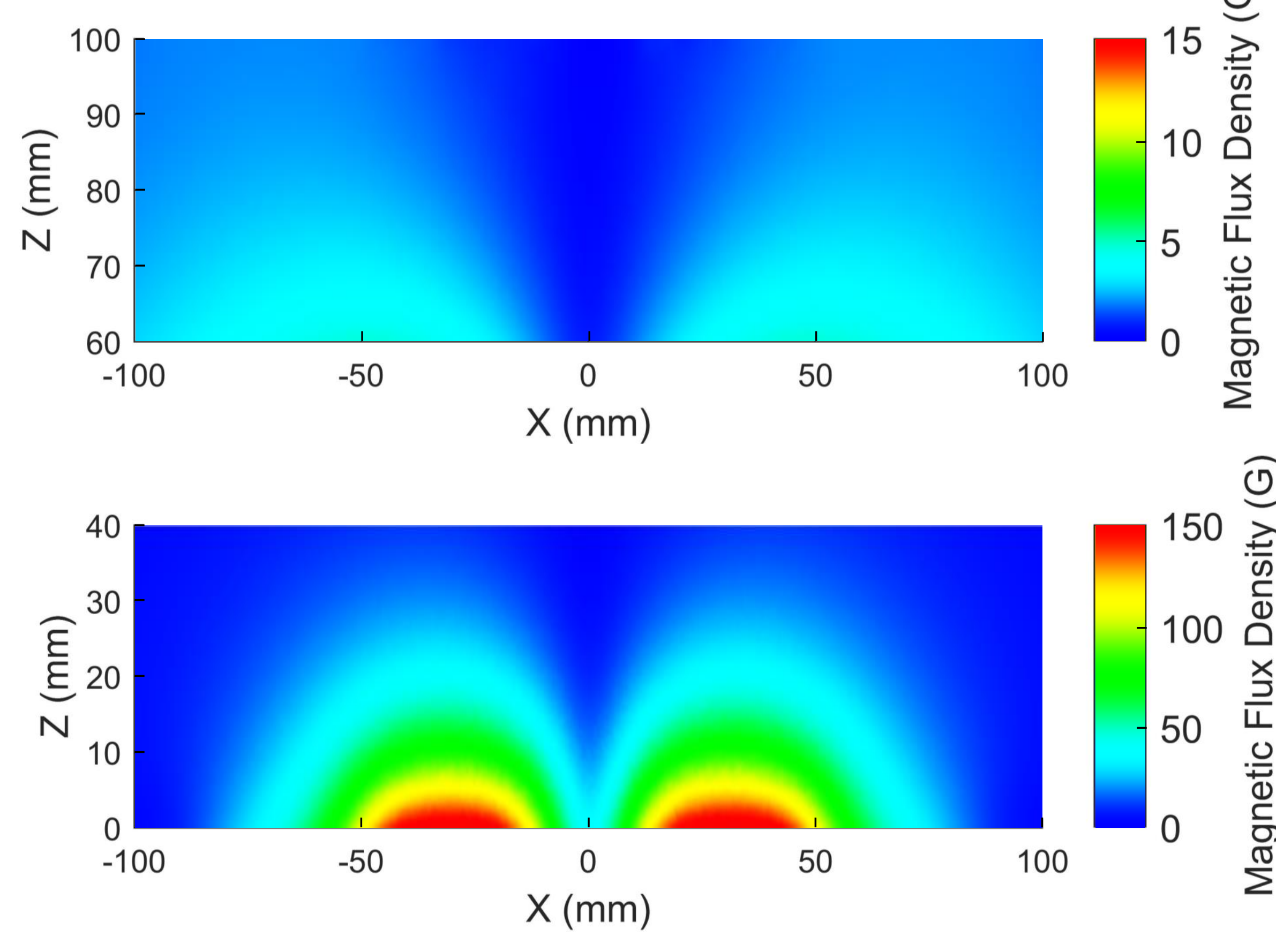

(b) 
Vacuum chamber

\begin{tabular}{ll} 
BS - beam splitters & $\mathrm{L}-$ lenses \\
$\mathrm{D}-$ diaphragm & $\mathrm{F} 1$ - shortpass filter \\
F2 - bandpass filter & \\
\hline
\end{tabular}

window1

window2
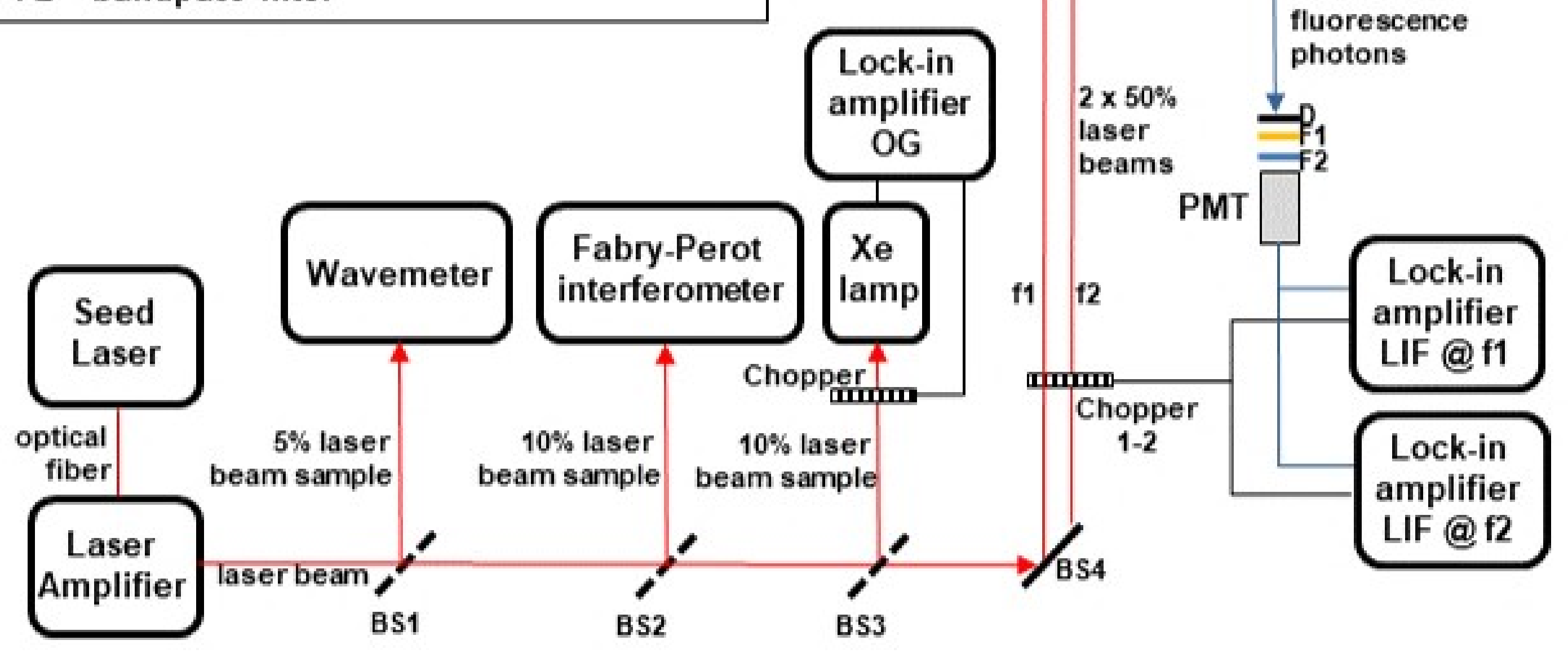


\section{Vacuum chamber}

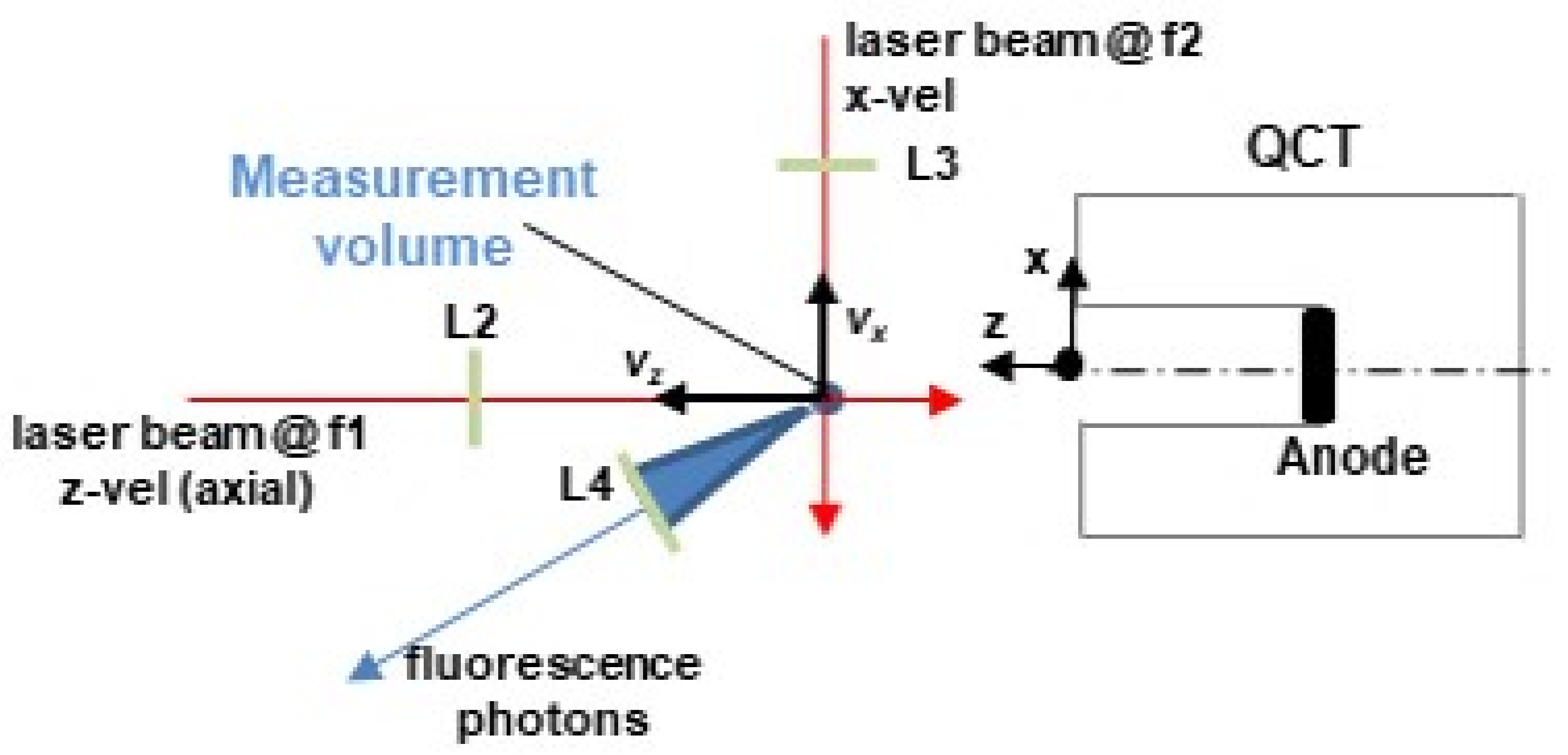


(a) Axial Velocity

$(\mathrm{km} / \mathrm{s})$
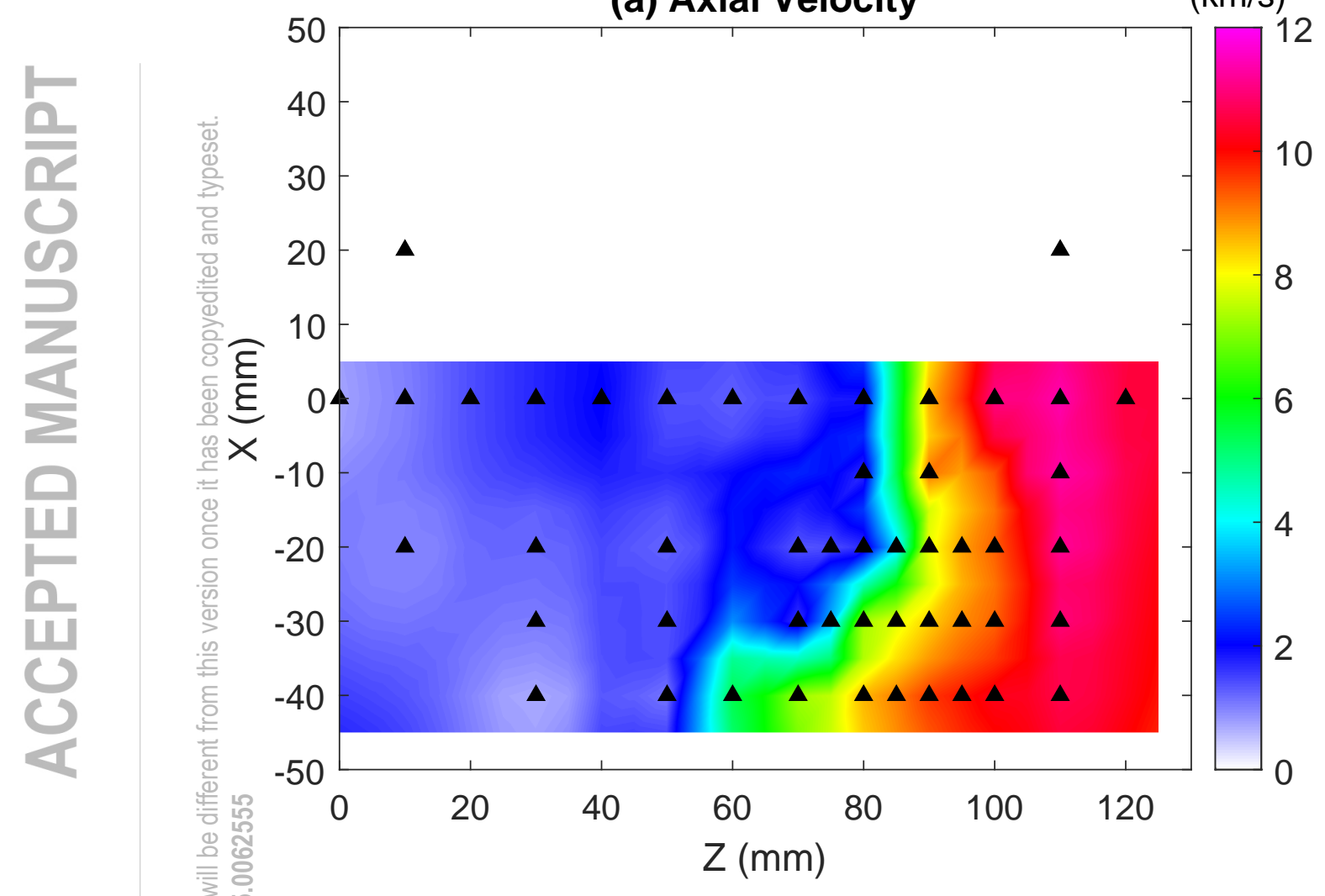
(b) Transverse Velocity

$(\mathrm{km} / \mathrm{s})$
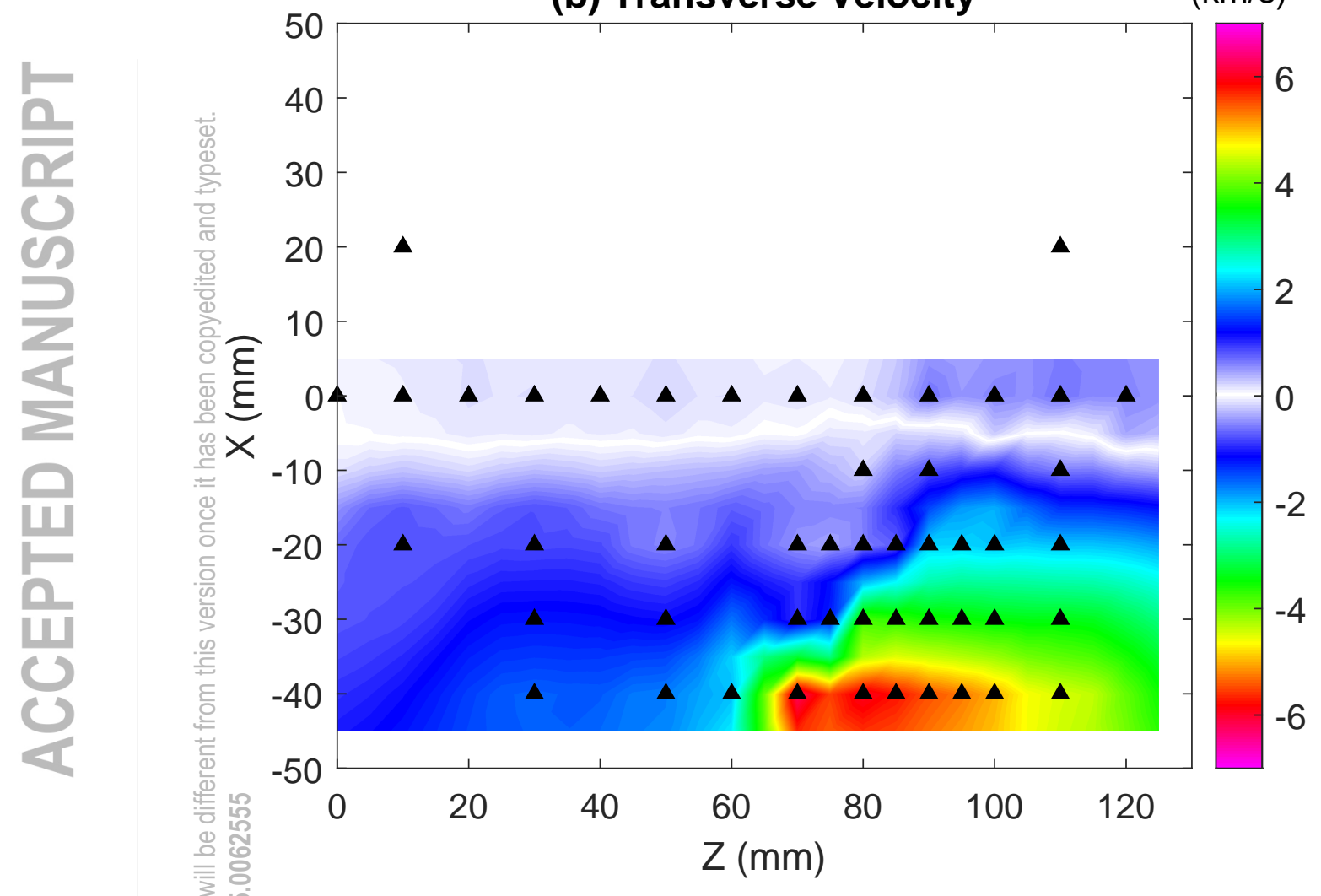
(c) Velocity Angle From Z-Axis

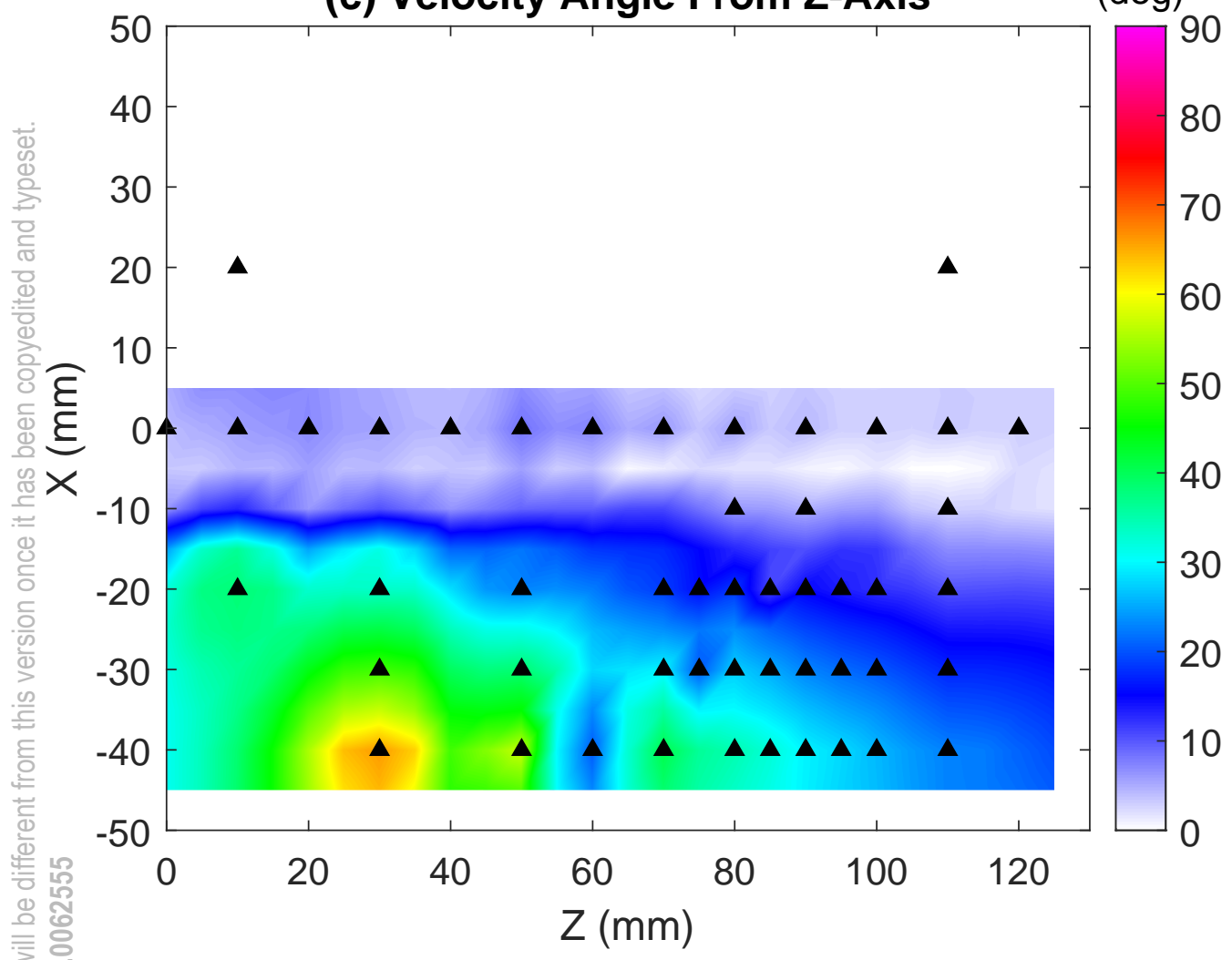


(d) Velocity Vectors

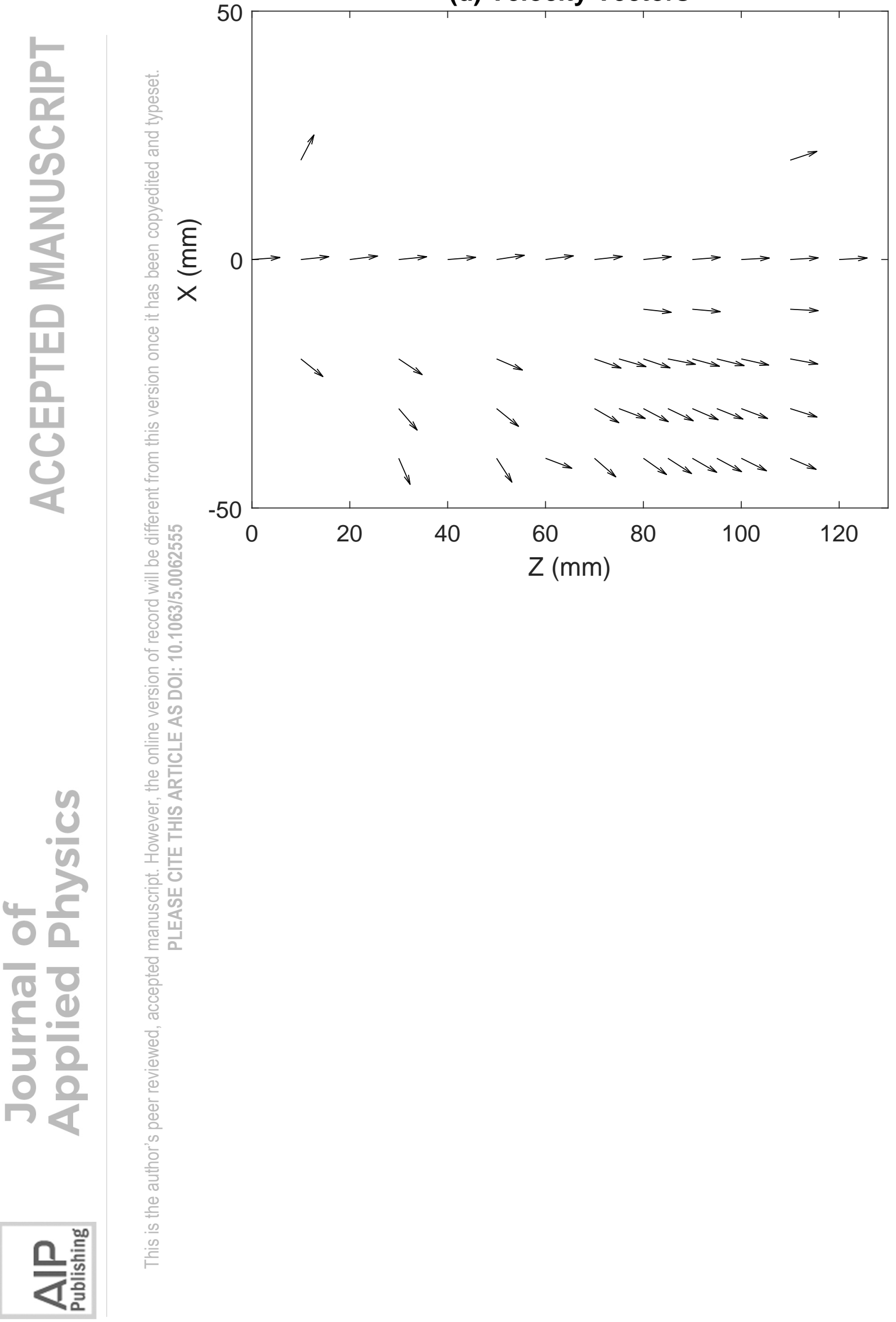




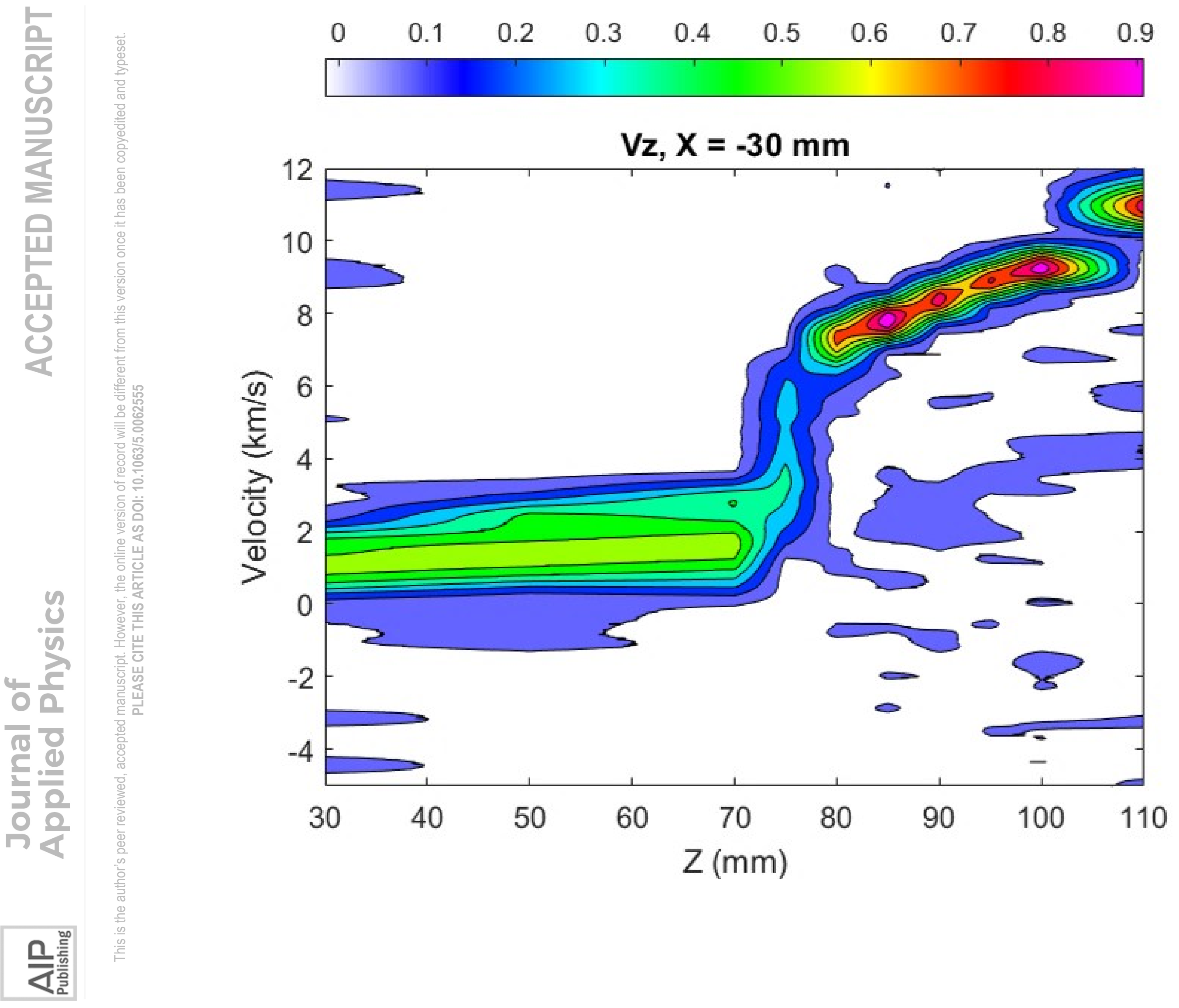




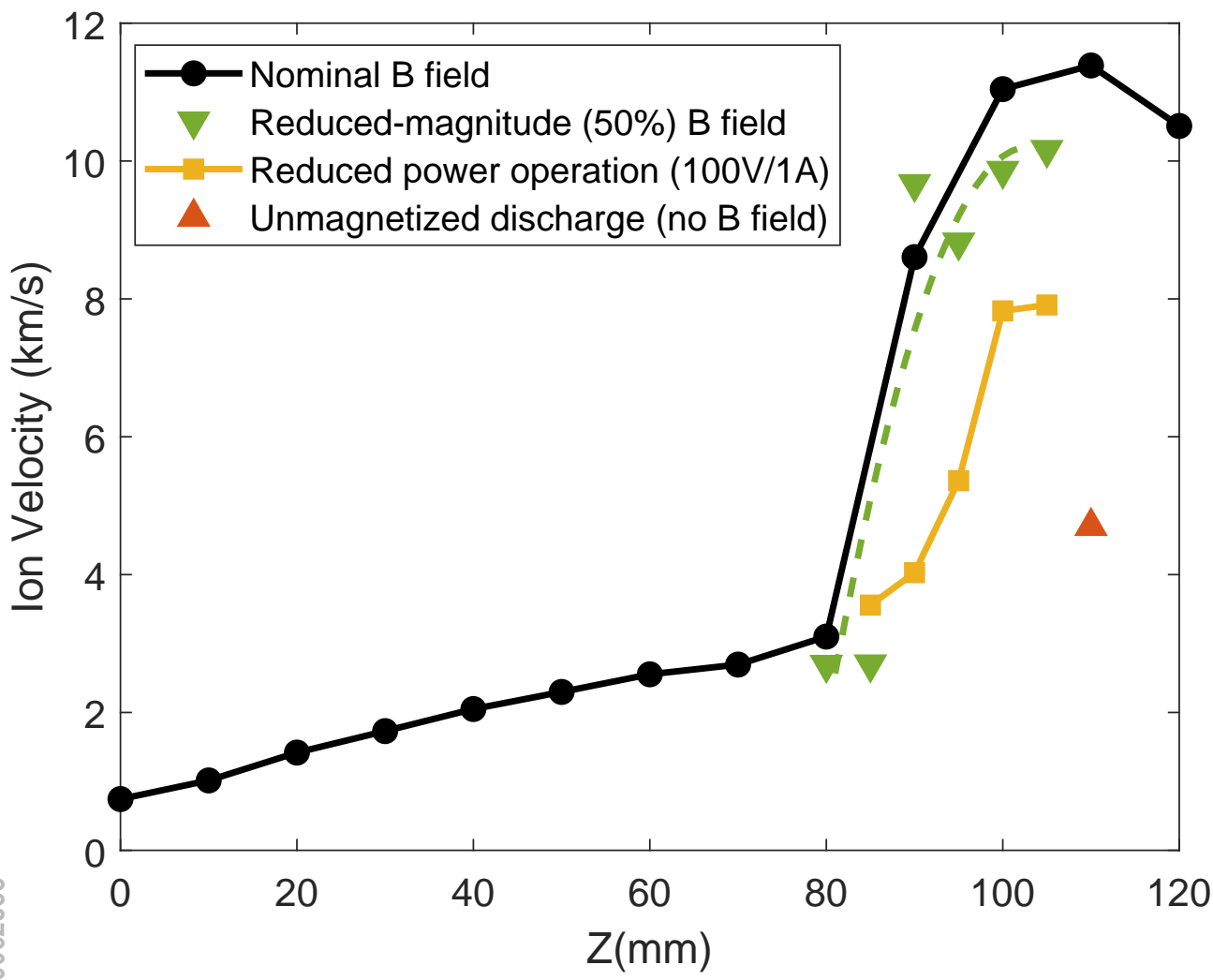




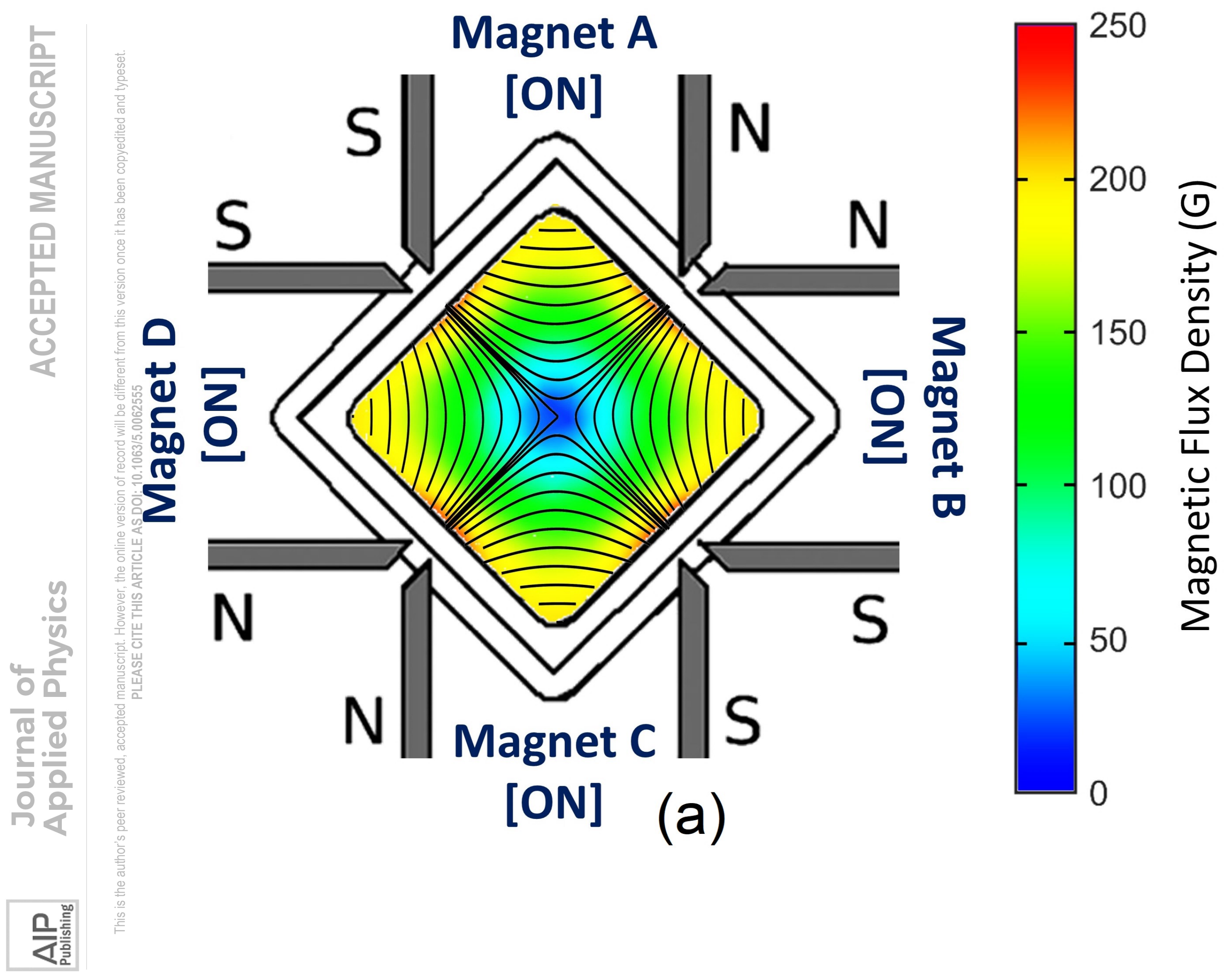




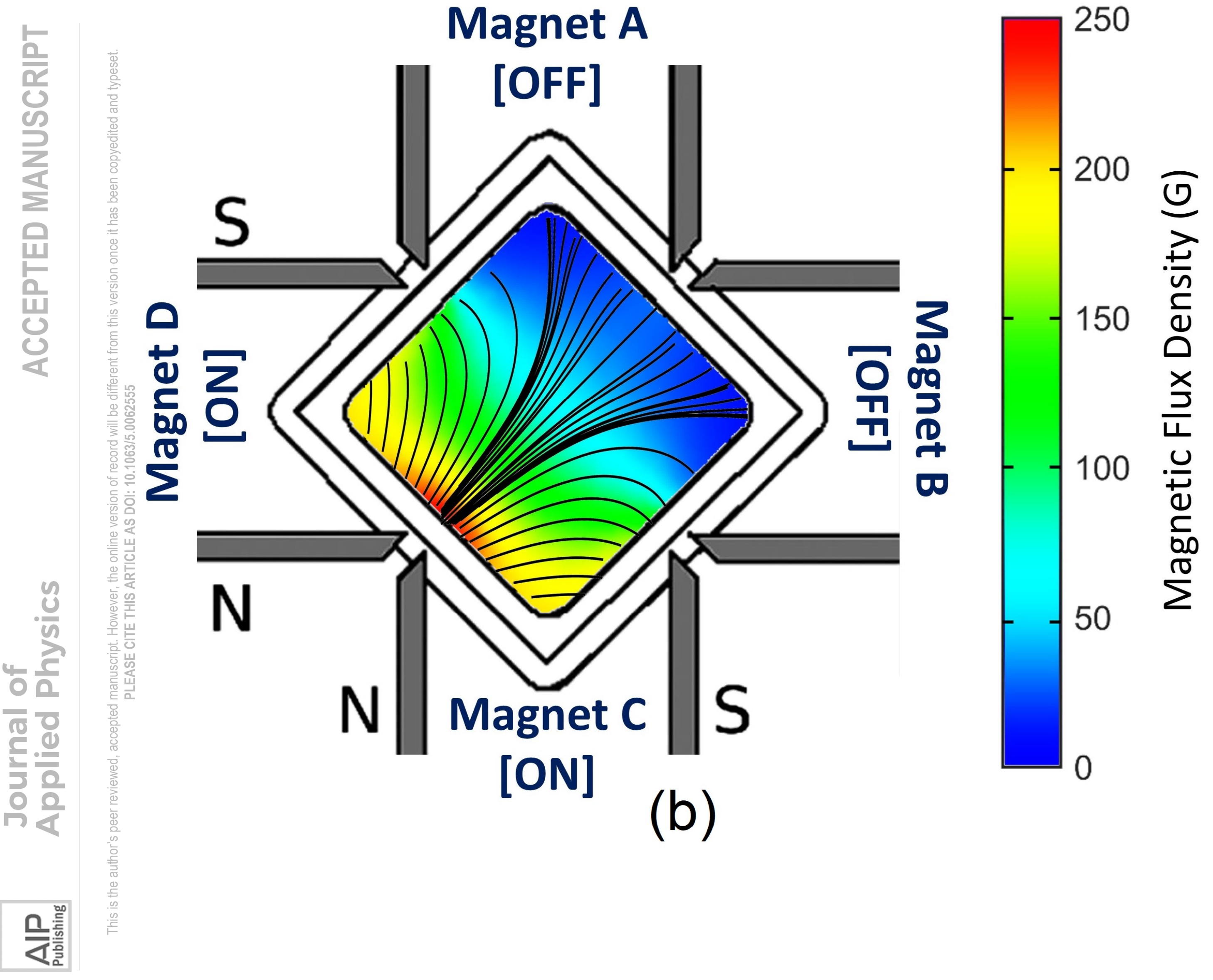


(a) Axial Velocity

$(\mathrm{km} / \mathrm{s})$

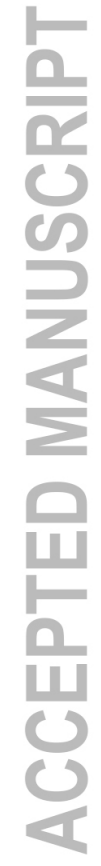

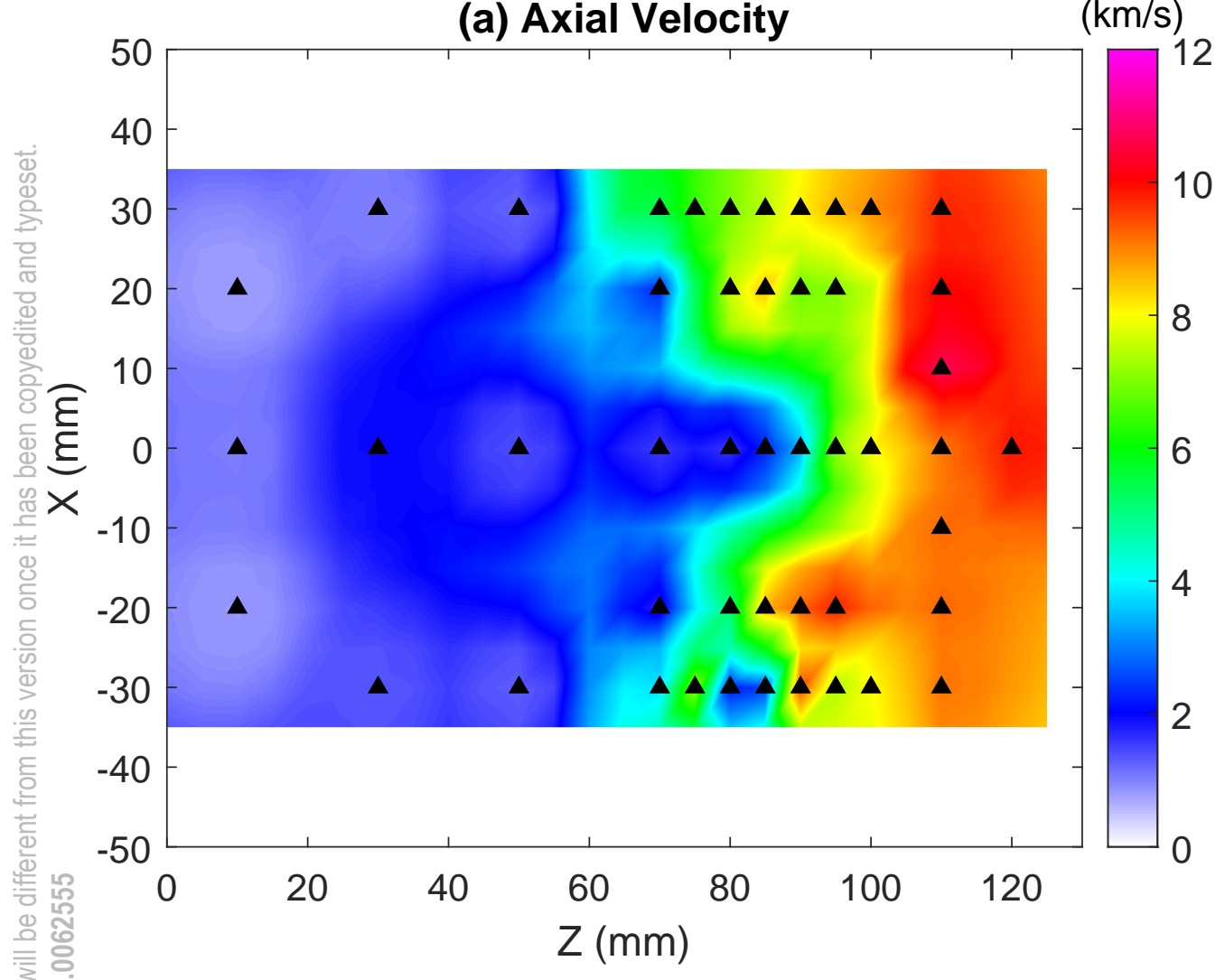


(b) Transverse Velocity $(\mathrm{km} / \mathrm{s})$

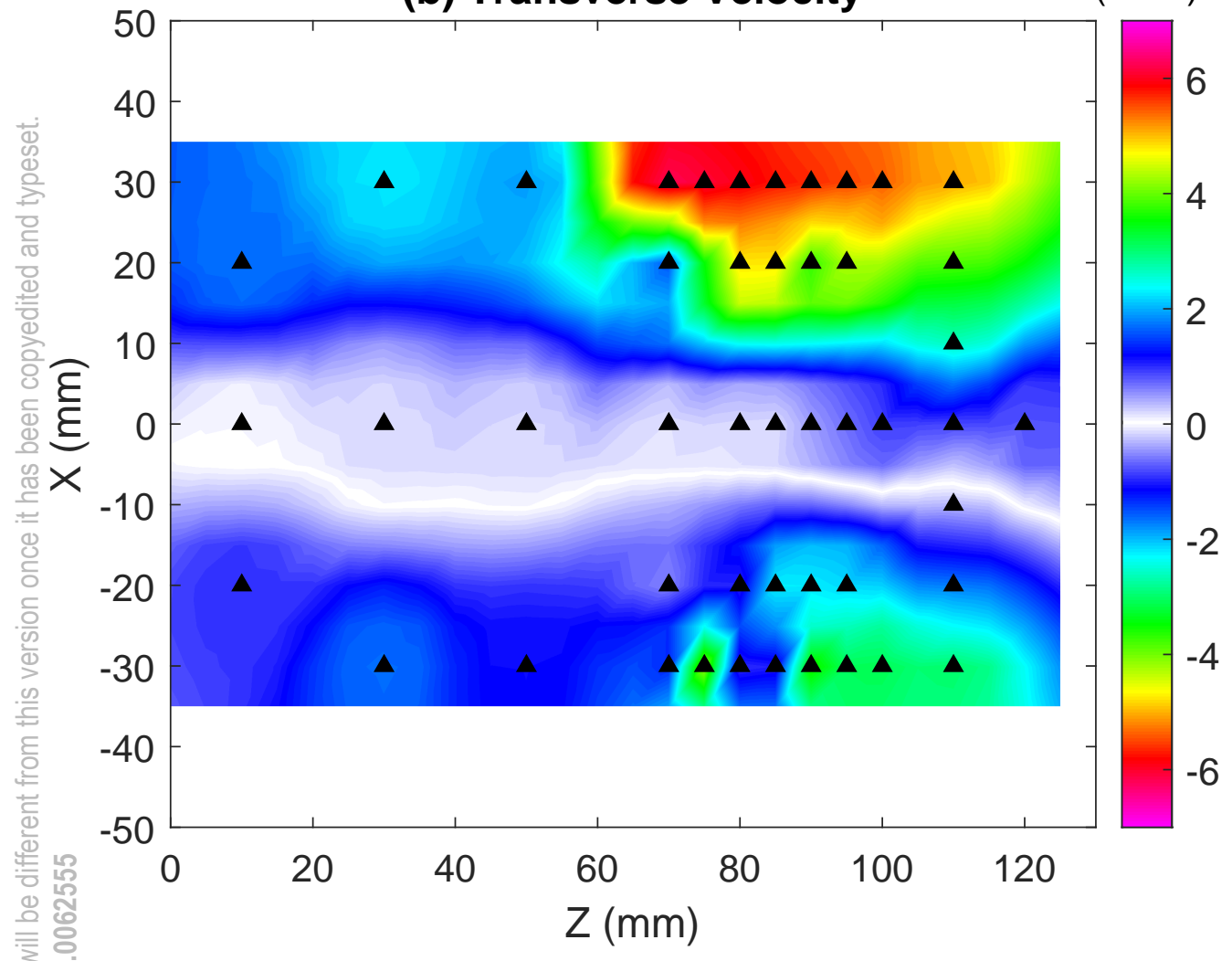


(c) Velocity Angle From Z-Axis (deg)

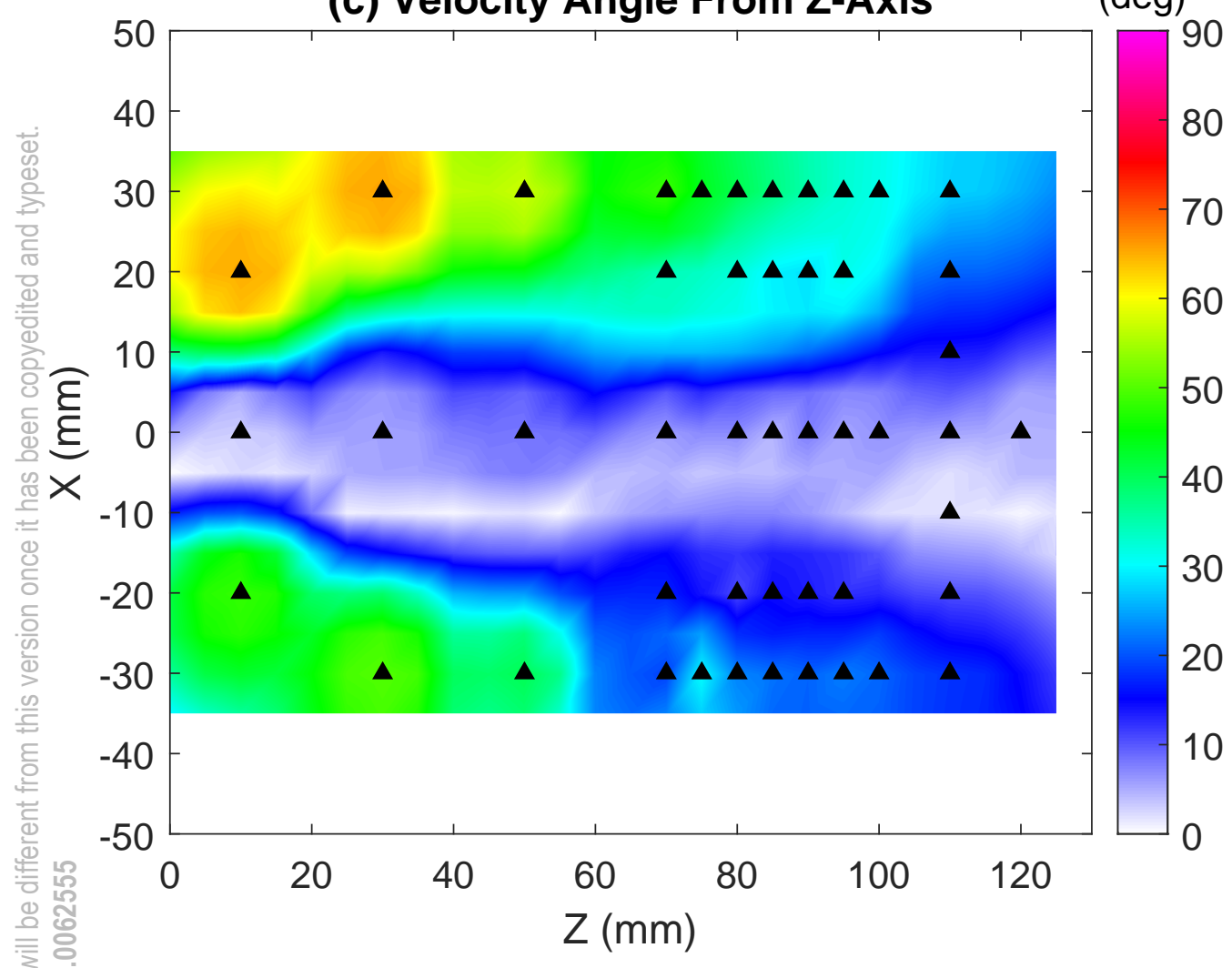


(d) Velocity Vectors

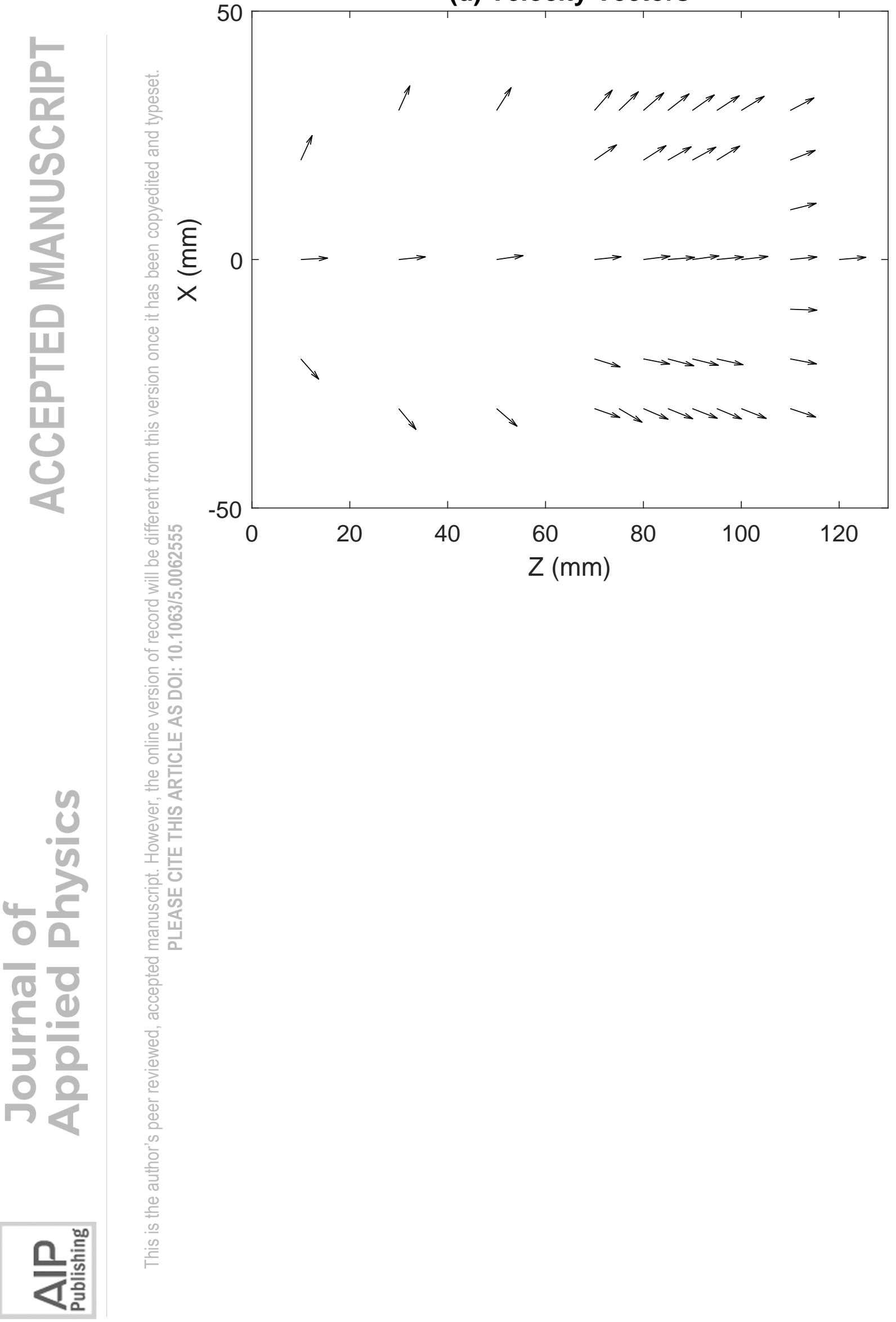



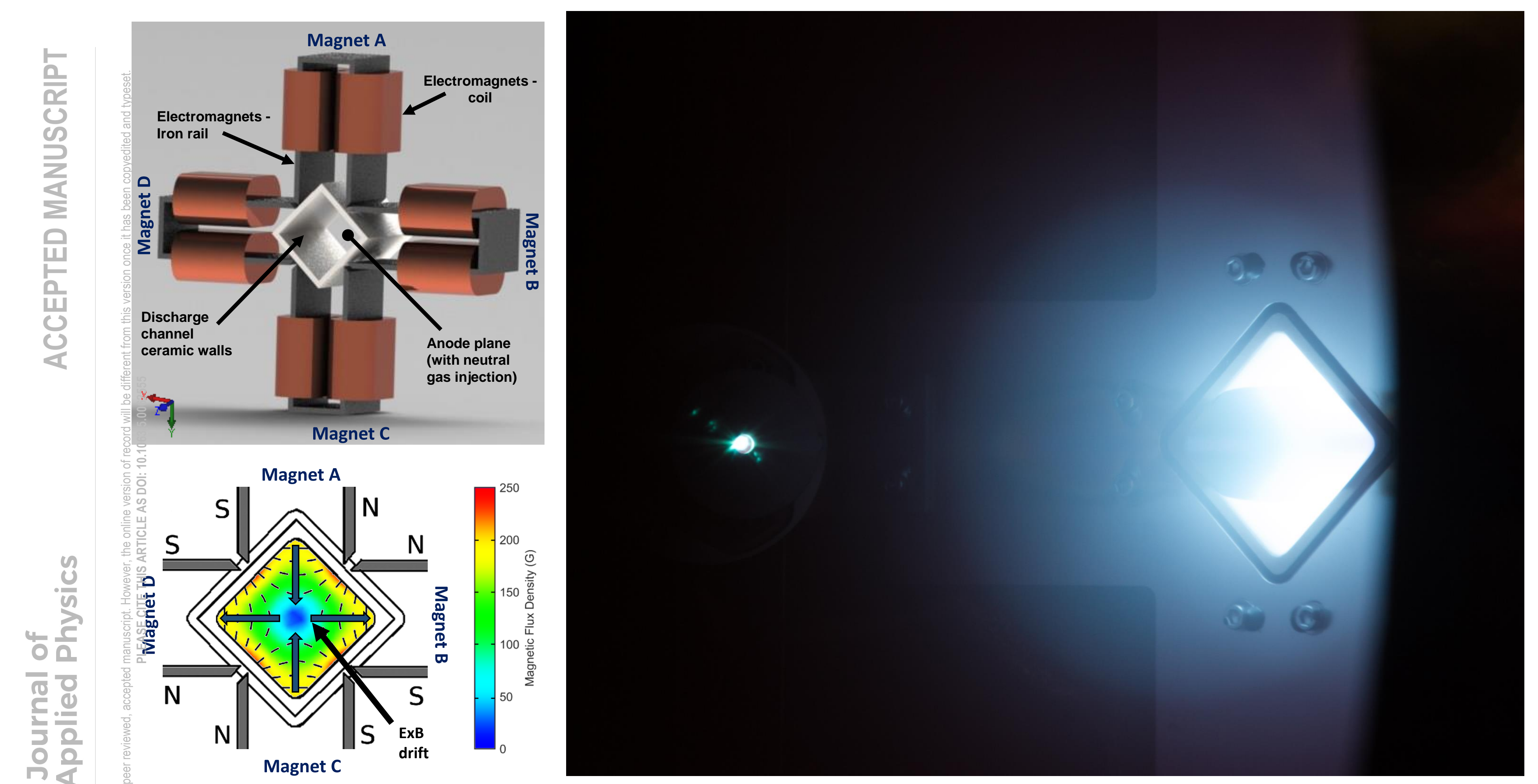

매의 